\title{
Solenoidal difference quotients and their application to the regularity theory of the $p$-Stokes system
}

\author{
Martin Křepela ${ }^{1} \cdot$ Michael Růžička ${ }^{1}$
}

Received: 4 April 2019 / Accepted: 30 November 2019 / Published online: 18 January 2020

(c) The Author(s) 2020

\begin{abstract}
We prove existence of a solution to the divergence equation satisfying a new Bogovski-type estimate for the difference quotients. This enables us to give an alternative proof of the interior regularity of the solution to the $p$-Stokes problem, completely avoiding the pressure. Moreover, as a key preliminary result we prove boundedness of Calderón-Zygmund operators with standard kernels in weighted Lebesgue and Orlicz spaces over a general domain.
\end{abstract}

Mathematics Subject Classification 42B20 $\cdot 42 \mathrm{~B} 37 \cdot 35 \mathrm{~B} 65 \cdot 35 \mathrm{~B} 45 \cdot 35 \mathrm{~J} 92$

\section{Introduction}

We are concerned with the question of interior regularity of the weak solution of the steady Stokes approximation for flows of shear thinning fluids. It is given by

$$
\begin{aligned}
-\operatorname{div} \mathbf{S}(\mathbf{D u})+\nabla \pi=\mathbf{f} & \text { in } \Omega, \\
\operatorname{div} \mathbf{u}=0 & \text { in } \Omega, \\
\mathbf{u}=\mathbf{0} & \text { on } \partial \Omega,
\end{aligned}
$$

where $\Omega \subset \mathbb{R}^{n}, n \geq 2$, is a bounded domain. In here, $\mathbf{u}=\left(u^{1}, \ldots, u^{n}\right)^{\top}$ denotes the unknown velocity vector field, and $\pi$ the unknown scalar pressure, while the external body force $\mathbf{f}=\left(f^{1}, \ldots, f^{n}\right)^{\top}$ is given. The extra stress tensor $\mathbf{S}$ depends only on $\mathbf{D u}:=\frac{1}{2}(\nabla \mathbf{u}+$ $\nabla \mathbf{u}^{\top}$ ), the symmetric part of the velocity gradient $\nabla \mathbf{u}$. The relevant example we have in mind is

$$
\mathbf{S}(\mathbf{D u})=\mu(\delta+|\mathbf{D u}|)^{p-2} \mathbf{D u},
$$

Communicated by Y. Giga.

$\bowtie$ Michael Růžička

rose@mathematik.uni-freiburg.de

Martin Křepela

martin.krepela@math.uni-freiburg.de

1 Department of Applied Mathematics, University of Freiburg, Ernst-Zermelo-Straße 1, 79104 Freiburg, Germany 
with $p \in(1,2], \delta \geq 0$, and $\mu>0$. Notice that despite various efforts the optimal global regularity of this problem is still open (see $[1,4,8]$ for partial results). However, the interior regularity we focus on here is well-known (see $[4,15,19])$. The standard proof uses localized difference quotients in each direction. Due to the localization, the corresponding test function is not solenoidal anymore, therefore appropriate properties of the pressure have to be used.

Here we modify this approach and use a solenoidal version of the localized difference quotient. Thus, we can completely avoid the pressure $\pi$. This is a completely new approach even for the classical Stokes problem, i.e., $p=2$ in (1.2). To make it possible, we show that the solution of the divergence equation obtained via the Bogovski formula (see $[5,6]$ ) satisfies an additional estimate for the difference quotient. The proof of this is based on estimates of singular Calderón-Zygmund operators generated by standard kernels in arbitrary domains $\Omega \subset \mathbb{R}^{n}$ (see Theorem 3.4). This result is of independent interest since it shows that an analogy of the classical Calderón-Zygmund estimates (cf. [7,18]) in the whole space also hold in arbitrary domains. To prove it, we employ ideas from [5,6,10,16,23], where the divergence equation is treated, and modify them for our purposes. An important feature of our result is a careful tracking of the dependence of the constants on various quantities, which is missing in the literature.

\section{Preliminaries}

\subsection{Notation}

Throughout the text, we use the symbols $C, c$ to denote generic constants which may change from line to line but are independent of "crucial" quantities. In many cases, the dependence of such constants on various quantities will be explicitly specified. Furthermore, we write $f \sim g$ if there exist constants $c, C>0$ such that $c f \leq g \leq C f$.

A set $Q \subset \mathbb{R}^{n}$ is called an (open) cube if there exist $a_{1}, \ldots, a_{n} \in \mathbb{R}$ and $\ell(Q)>0$ such that

$$
Q=\left\{\mathbf{x} \in \mathbb{R}^{n} \mid a_{i}<x_{i}<a_{i}+\ell(Q), i=1, \ldots, n\right\} .
$$

The number $\ell(Q)$ is the side length of $Q$ and the point $\mathbf{c}:=\left(a_{1}+\frac{1}{2} \ell(Q), \ldots, a_{n}+\frac{1}{2} \ell(Q)\right)^{\top}$ is the center of the cube $Q$, which can be also denoted by $Q=Q(\mathbf{c}, \ell(Q))$. For $\alpha>0$ and a cube $Q=Q(\mathbf{c}, \ell(Q))$ we use the notation $\alpha Q:=Q(\mathbf{c}, \alpha \ell(Q))$. Moreover, a cube $Q$ is said to be dyadic if there exist $j_{i}, \ldots, j_{n}, k \in \mathbb{Z}$ such that

$$
Q=\left\{\mathbf{x} \in \mathbb{R}^{n} \mid j_{i} 2^{k}<x_{i}<\left(j_{i}+1\right) 2^{k}, i=1, \ldots, n\right\} .
$$

Notice that, in the above definition, we consider only cubes with faces parallel to the coordinate axes.

While working with function spaces, we do not distinguish between spaces of scalar, vector-valued or tensor-valued functions. However, we denote vectors by boldface lower-case letters (e.g., u) and tensors by boldface upper-case letters (e.g., $\mathbf{S})$. For vectors $\mathbf{u}, \mathbf{v} \in \mathbb{R}^{n}$, the standard tensor product $\mathbf{u} \otimes \mathbf{v} \in \mathbb{R}^{n \times n}$ is defined as $(\mathbf{u} \otimes \mathbf{v})_{i j}:=u_{i} v_{j}$, and the symmetric tensor product as $\mathbf{u} \stackrel{s}{\otimes} \mathbf{v}:=\frac{1}{2}\left(\mathbf{u} \otimes \mathbf{v}+(\mathbf{u} \otimes \mathbf{v})^{\top}\right)$. The scalar product of vectors is denoted by $\mathbf{u} \cdot \mathbf{v}=\sum_{i=1}^{n} u_{i} v_{i}$ and the scalar product of tensors is denoted by $\mathbf{A} \cdot \mathbf{B}:=\sum_{i, j=1}^{n} A_{i j} B_{i j}$.

If $E \subset \mathbb{R}^{n}$ is a measurable set, $|E|$ denotes its Lebesgue measure. In the following definitions of function spaces, we always assume $E$ to be a domain in $\mathbb{R}^{n}$, with a sufficiently smooth boundary, if needed. We use standard Lebesgue spaces $\left(L^{p}(E),\|\cdot\|_{p}\right)$ and Sobolev 
spaces $\left(W^{k, p}(E),\|\cdot\|_{k, p}\right)$. If the underlying domain $E$ needs to be indicated, we denote the respective norms by $\|\cdot\|_{L^{p}(E)}$ and $\|\cdot\|_{W^{k, p}(E)}$. If $p \in(1, \infty)$, we denote by $p^{\prime}$ the conjugated exponent $p^{\prime}:=\frac{p}{p-1}$.

Besides the standard $L^{p}$ spaces we will also consider their weighted variants. A weight is any measurable function $\omega: \mathbb{R}^{n} \rightarrow[0, \infty)$. If $p \in[1, \infty)$ and $\omega$ is a weight, the space $L_{\omega}^{p}(E)$ consists of all measurable functions $f$ on $E$ such that

$$
\|f\|_{L_{\omega}^{p}(E)}:=\left(\int_{E}|f(\mathbf{x})|^{p} \omega(\mathbf{x}) \mathrm{d} \mathbf{x}\right)^{\frac{1}{p}}<\infty .
$$

If $p>1$, an $A_{p}$ weight is a weight such that $\omega$ and $\omega^{1-p^{\prime}}$ are locally integrable and

$$
[\omega]_{A_{p}}:=\sup _{B \subset \mathbb{R}^{n}} \frac{1}{|B|} \int_{B} \omega(\mathbf{x}) \mathrm{d} \mathbf{x}\left(\frac{1}{|B|} \int_{B} \omega^{1-p^{\prime}}(\mathbf{x}) \mathrm{d} \mathbf{x}\right)^{p-1}<\infty,
$$

where $B \subset \mathbb{R}^{n}$ are balls. In this case, we write $\omega \in A_{p}$. If $p=1$, a weight is called an $A_{1}$ weight if $0<\omega<\infty$ a.e. and

$$
[\omega]_{A_{1}}:=\operatorname{esssup}_{x \in \mathbb{R}^{n}} \frac{M \omega(\mathbf{x})}{\omega(\mathbf{x})}<\infty .
$$

In here, $M$ is the maximal operator (with respect to non-centered balls), defined by

$$
M \omega(\mathbf{x}):=\sup _{B \ni \mathbf{x}} \frac{1}{|B|} \int_{B}|\omega(\mathbf{y})| \mathrm{d} \mathbf{y}
$$

for $\mathbf{x} \in \mathbb{R}^{n}$, where $B \ni \mathbf{x}$ means a ball containing $\mathbf{x}$. Notice that $[\omega]_{A_{p}} \geq 1$ for all weights $\omega$ and all $p \in[1, \infty)$, and the identity holds if and only if $\omega$ is constant (see [18, Proposition 7.1.5]).

The symbol supp $f$ denotes the support of a function $f$. The set of all compactly supported, smooth functions defined on $E$ is denoted by $C_{0}^{\infty}(E)$. The space $W_{0}^{1, p}(E)$ is the closure of $C_{0}^{\infty}(E)$ in $W^{1, p}(E)$. If $E$ is bounded, the space $W_{0}^{1, p}(E)$ may be equipped with the gradient norm $\|\nabla \cdot\|_{p}$, thanks to the Poincaré inequality. Next, we denote by $W_{0 \text {,div }}^{1, p}(E)$ the subspace of $W_{0}^{1, p}(E)$ consisting of solenoidal vector fields $\mathbf{u}$, i.e., such that $\operatorname{div} \mathbf{u}=0$. By $L_{\omega, 0}^{p}(E)$ we denote the subspace of $L_{\omega}^{p}(E)$ consisting of functions $f$ with vanishing mean value, i.e., such that $\int_{E} f(\mathbf{x}) \mathrm{d} \mathbf{x}=0$.

Orlicz and Sobolev-Orlicz spaces also appear frequently in this article. We briefly present their elementary properties here, for details we refer to [20,21].

An $N$-function is a continuous, nonnegative, strictly increasing and convex function $\psi$ on $[0, \infty)$ which additionally satisfies $\psi(0)=0, \lim _{t \rightarrow \infty} \frac{\psi(t)}{t}=\infty$ and $\lim _{t \rightarrow 0+} \frac{\psi(t)}{t}=0$. Its conjugate $N$-function $\psi^{*}:[0, \infty) \rightarrow[0, \infty)$ is then defined by

$$
\psi^{*}(t):=\sup _{s \geq 0}(s t-\psi(s))
$$

Furthermore, we define

$$
\Delta_{2}(\psi):=\sup \left\{\frac{\psi(2 t)}{\psi(t)} \mid t \in[0, \infty)\right\} .
$$

If $\Delta_{2}(\psi)<\infty$, we say that $\psi$ satisfies the $\Delta_{2}$-condition. From now on, let us assume that $\Delta_{2}(\psi)<\infty$ and $\Delta_{2}\left(\psi^{*}\right)<\infty$. Then we denote by $L^{\psi}(E)$ and $W^{1, \psi}(E)$ the classical Orlicz and Sobolev-Orlicz spaces, respectively. More precisely, $f \in L^{\psi}(E)$ if the modular 


$$
\varrho_{\psi}(f):=\int_{E} \psi(|f(\mathbf{x})|) \mathrm{d} \mathbf{x}
$$

is finite, and $f \in W^{1, \psi}(E)$ if both $f$ and $\nabla f$ belong to $L^{\psi}(E)$. When equipped with the Luxemburg norm

$$
\|f\|_{\psi}:=\inf \left\{\lambda>0 \mid \int_{E} \psi(|f(\mathbf{x})| / \lambda) \mathrm{d} \mathbf{x} \leq 1\right\}
$$

the space $L^{\psi}(E)$ becomes a Banach space. The same holds for the space $W^{1, \psi}(E)$ when equipped with the norm $\|\cdot\|_{\psi}+\|\nabla \cdot\|_{\psi}$. Notice that the dual space $\left(L^{\psi}(E)\right)^{*}$ can be identified with the space $L^{\psi^{*}}(E)$. Furthermore, by $W_{0}^{1, \psi}(E)$ we denote the closure of $C_{0}^{\infty}(E)$ in $W^{1, \psi}(E)$. If $E$ is bounded and sufficiently regular, the Poincaré inequality for Orlicz modulars (see [25, Lemma 3]) implies that $W_{0}^{1, \psi}(E)$ may be equipped with the gradient norm $\|\nabla \cdot\|_{\psi}$. By $L_{0}^{\psi}(E)$ and $C_{0,0}^{\infty}(E)$ we denote the subspaces of $L^{\psi}(E)$ and $C_{0}^{\infty}(E)$, respectively, consisting of functions $f$ such that $\int_{E} f(\mathbf{x}) \mathrm{d} \mathbf{x}=0$.

If $\psi$ is an $N$-function satisfying $\Delta_{2}(\psi)<\infty$ and $\Delta_{2}\left(\psi^{*}\right)<\infty$, then for all $\varepsilon>0$ there exists a constant $c_{\varepsilon}>0$ such that

$$
t s \leq \varepsilon \psi(t)+c_{\varepsilon} \psi^{*}(s)
$$

holds for all $s, t \geq 0$, and

$$
t \psi^{\prime}(s)+\psi^{\prime}(t) s \leq \varepsilon \psi(t)+c_{\varepsilon} \psi(s)
$$

holds for a.e. $s, t>0$.

\subsection{Difference quotient}

If $k \in\{1, \ldots, n\}$, we denote by $\mathbf{e}^{k}$ the $k$-th vector of the canonical basis of the Euclidean space $\mathbb{R}^{n}$, i.e., $\mathbf{e}^{k}=\left(\delta_{i k}\right)_{i=1}^{n}$. If $E \subset \mathbb{R}^{n}$, we denote

$$
\begin{aligned}
E \pm h \mathbf{e}^{k} & :=\left\{\mathbf{x} \in \mathbb{R}^{n} \mid \exists \mathbf{y} \in E: \mathbf{x}=\mathbf{y} \pm h \mathbf{e}^{k}\right\}, \\
E_{h} & :=\{\mathbf{x} \in E \mid \operatorname{dist}(\mathbf{x}, \partial E)>h\} .
\end{aligned}
$$

Let $\mathbf{F}: \mathbb{R}^{n} \rightarrow \mathbb{R}^{n \times n}$ be a measurable tensor field (or a vector field or a real-valued function) and $h>0$. Then we define the difference quotients of $\mathbf{F}$ as follows:

$$
d_{h, k}^{ \pm} \mathbf{F}(\mathbf{x}):=\frac{\mathbf{F}\left(\mathbf{x} \pm h \mathbf{e}^{k}\right)-\mathbf{F}(\mathbf{x})}{h}, \quad \mathbf{x} \in \mathbb{R}^{n} .
$$

We will also use the notation $\Delta_{h, k}^{ \pm} \mathbf{F}(\mathbf{x}):=h d_{h, k}^{ \pm} \mathbf{F}(\mathbf{x})$. It is well-known (cf. [14, Sect. 5.8]) that for $\mathbf{F} \in W^{1,1}\left(\mathbb{R}^{n}\right)$ one has

$$
\lim _{h \rightarrow 0+} d_{h, k}^{ \pm} \mathbf{F}(\mathbf{x})=\partial_{k} \mathbf{F}(\mathbf{x}) \text { for a.e. } \mathbf{x} \in \mathbb{R}^{n},
$$

and

$$
\nabla d_{h, k}^{ \pm} \mathbf{F}(\mathbf{x})=d_{h, k}^{ \pm} \nabla \mathbf{F}(\mathbf{x}) \text { for a.e. } \mathbf{x} \in \mathbb{R}^{n} .
$$

Elementary calculations show that we have the following variant of the product rule for $\mathbf{F}, \mathbf{G} \in L_{\mathrm{loc}}^{1}\left(\mathbb{R}^{n}\right)$

$$
d_{h, k}^{ \pm}(\mathbf{F} \mathbf{G})(\mathbf{x})=\mathbf{F}\left(\mathbf{x} \pm h \mathbf{e}^{k}\right) d_{h, k}^{ \pm} G(\mathbf{x})+d_{h, k}^{ \pm} \mathbf{F}(\mathbf{x}) \mathbf{G}(\mathbf{x}) \text { for a.e. } \mathbf{x} \in \mathbb{R}^{n} .
$$


For $\mathbf{F}, \mathbf{G} \in L^{1}(E)$, extended by zero outside $E$, the partial integration formula

$$
\int_{E} \mathbf{F} d_{h, k}^{+} \mathbf{G} \mathrm{d} \mathbf{x}=\int_{E} d_{h, k}^{-} \mathbf{F ~} \mathbf{G} \mathrm{d} \mathbf{x}
$$

holds. Moreover, for every $h_{0}>0$, every open set $E \subset \mathbb{R}^{n}$, every $\mathbf{F} \in W_{\text {loc }}^{1, \psi}\left(\mathbb{R}^{n}\right)$ and all $h \leq h_{0}$ we have

$$
\int_{E_{h_{0}}} \psi\left(\left|d_{h, k}^{ \pm} \mathbf{F}(\mathbf{x})\right|\right) \mathrm{d} \mathbf{x} \leq \int_{E} \psi\left(\left|\partial_{k} \mathbf{F}(\mathbf{x})\right|\right) \mathrm{d} \mathbf{x} .
$$

The proof of this assertion in the case of the special $N$-function $\psi(t)=t^{p}$ can be found in [14, Theorem 3 (i) in Section 5.8]). In fact, replacing the special $N$-function $t^{p}$ by a general $N$-function $\psi$ one can proceed exactly as outlined there to obtain (2.4). These comments also apply for the converse statement [14, Theorem 3 (ii) in Section 5.8]). More precisely, if $d_{h, k}^{ \pm} \mathbf{F} \in L^{\psi}\left(E_{h_{0}}\right)$ for all $h_{0}>0$ and all $0<h<h_{0}$ satisfy

$$
\int_{E_{h_{0}}} \psi\left(\left|d_{h, k}^{ \pm} \mathbf{F}(\mathbf{x})\right|\right) \mathrm{d} \mathbf{x} \leq c_{1},
$$

then $\partial_{k} \mathbf{F}$ exists in the sense of distributions and satisfies

$$
\int_{E} \psi\left(\left|\partial_{k} \mathbf{F}(\mathbf{x})\right|\right) \mathrm{d} \mathbf{x} \leq c_{1}
$$

\subsection{Operators with kernels}

A measurable function $K: \mathbb{R}^{n} \times \mathbb{R}^{n} \rightarrow \mathbb{R}$ is called a kernel.

Definition 2.1 Let $E \subset \mathbb{R}^{n}$ be a domain. A kernel $K: \mathbb{R}^{n} \times \mathbb{R}^{n} \rightarrow \mathbb{R}$ is called a standard kernel with respect to $E$ if there exists a constant $\kappa_{1}>0$ such that, for any $\mathbf{x}, \mathbf{y}, \mathbf{z} \in E$ satisfying

$$
\mathbf{x} \neq \mathbf{y} \text { and }|\mathbf{x}-\mathbf{z}| \leq \frac{1}{2}|\mathbf{x}-\mathbf{y}|,
$$

the following conditions hold true:

$$
\begin{aligned}
&|K(\mathbf{x}, \mathbf{y})| \leq \frac{\kappa_{1}}{|\mathbf{x}-\mathbf{y}|^{n}}, \\
&|K(\mathbf{x}, \mathbf{y})-K(\mathbf{z}, \mathbf{y})| \leq \kappa_{1} \frac{|\mathbf{x}-\mathbf{z}|}{|\mathbf{x}-\mathbf{y}|^{n+1}}, \\
&|K(\mathbf{y}, \mathbf{x})-K(\mathbf{y}, \mathbf{z})| \leq \kappa_{1} \frac{|\mathbf{x}-\mathbf{z}|}{|\mathbf{x}-\mathbf{y}|^{n+1}} .
\end{aligned}
$$

The set of all kernels satisfying the above conditions with a given constant $\kappa_{1}$ will be denoted by $\operatorname{SK}\left(E, \kappa_{1}\right)$.

Remark 2.2 As it is common in the literature, it is possible to replace the right-hand side in (2.9) and (2.10) by

$$
\kappa_{1} \frac{|\mathbf{x}-\mathbf{z}|^{\delta}}{|\mathbf{x}-\mathbf{y}|^{n+\delta}}
$$

with $\delta>0$. Although the results could be obtained in this generalized setting as well, we restrict ourselves to the case $\delta=1$ to avoid further complications. 
Definition 2.3 We say that a linear operator $T$ on $C_{0}^{\infty}\left(\mathbb{R}^{n}\right)$ is generated by the kernel $K$ if

$$
T f(\mathbf{x})=\int_{\mathbb{R}^{n}} f(\mathbf{y}) K(\mathbf{x}, \mathbf{y}) \mathrm{d} \mathbf{y}
$$

holds whenever the right-hand side is well-defined. For a given kernel $K$ and $\varepsilon>0$ we define the truncated kernel $K_{\varepsilon}$ by

$$
K_{\varepsilon}(\mathbf{x}, \mathbf{y}):= \begin{cases}K(\mathbf{x}, \mathbf{y}) & \text { for }|\mathbf{x}-\mathbf{y}|>\varepsilon, \\ 0 & \text { for }|\mathbf{x}-\mathbf{y}| \leq \varepsilon,\end{cases}
$$

and denote by $T_{\varepsilon}$ the operator generated by the kernel $K_{\varepsilon}$.

Definition 2.4 Let $K$ be a kernel and let $E \subset \mathbb{R}^{n}$ be a domain. We call $K$ a CalderónZygmund kernel with respect to $E$, if there exists a constant $\kappa_{2} \in(0, \infty)$ such that the function $N: \mathbb{R}^{n} \times \mathbb{R}^{n} \rightarrow \mathbb{R}$, defined by

$$
N(\mathbf{x}, \mathbf{y}):=K(\mathbf{x}, \mathbf{x}-\mathbf{y}), \quad \mathbf{x}, \mathbf{y} \in \mathbb{R}^{n},
$$

satisfies the following conditions:

$$
\begin{aligned}
& N(\mathbf{x}, \alpha \mathbf{z})=\alpha^{-n} N(\mathbf{x}, \mathbf{z}) \text { for all } \mathbf{x}, \mathbf{z} \in \mathbb{R}^{n}, \quad \alpha>0 ; \\
& \int_{|\mathbf{z}|=1} N(\mathbf{x}, \mathbf{z}) \mathrm{d} \mathbf{z}=0 \text { for all } \mathbf{x} \in \mathbb{R}^{n} ; \\
& \sup _{\mathbf{x} \in E} \int_{|\mathbf{z}|=1}|N(\mathbf{x}, \mathbf{z})|^{2} \mathrm{~d} \mathbf{z} \leq \kappa_{2}^{2} .
\end{aligned}
$$

The set of all kernels satisfying the above conditions with a given constant $\kappa_{2}$ will be denoted by $\mathrm{CZ}\left(E, \kappa_{2}\right)$.

Remark 2.5 One can replace (2.14) by

$$
\sup _{\mathbf{x} \in E} \int_{|\mathbf{z}|=1}|N(\mathbf{x}, \mathbf{z})|^{q} \mathrm{~d} \mathbf{z} \leq \kappa_{2}^{q},
$$

where $q \in(1, \infty)$, and still retain the relevant properties of the operator generated by the kernel. For the sake of simplicity, we use the condition only with $q=2$.

\section{$2.4(p, \delta)$-structure}

Let us define what it means that a tensor field $\mathbf{S}$ has a $(p, \delta)$-structure. For details on this matter, see $[11,22]$. For a tensor $\mathbf{P} \in \mathbb{R}^{n \times n}$ we denote its symmetric part by

$$
\mathbf{P}^{\mathrm{sym}}:=\frac{\mathbf{P}+\mathbf{P}^{\top}}{2} \in \mathbb{R}_{\mathrm{sym}}^{n \times n},
$$

where

$$
\mathbb{R}_{\mathrm{sym}}^{n \times n}:=\left\{\mathbf{P} \in \mathbb{R}^{n \times n} \mid \mathbf{P}=\mathbf{P}^{\top}\right\} .
$$

We use the notation $|\mathbf{P}|^{2}=\mathbf{P} \cdot \mathbf{P}$.

It is convenient to define a special $N$-function $\varphi=\varphi_{p, \delta}$, with $p \in(1, \infty), \delta \geq 0$, by

$$
\varphi(t):=\int_{0}^{t}(\delta+s)^{p-2} s \mathrm{~d} s, \quad t \geq 0 .
$$


The function $\varphi$ satisfies, uniformly in $t$ and independently of $\delta$, the equivalences

$$
\varphi^{\prime \prime}(t) t \sim \varphi^{\prime}(t), \quad \varphi^{\prime}(t) t \sim \varphi(t), \quad t^{p}+\delta^{p} \sim \varphi(t)+\delta^{p} .
$$

In case that the (one-sided) derivative $\varphi^{\prime \prime}(0)$ does not exist, we assume that $\varphi^{\prime \prime}(t) t$ is continuously extended by zero for $t=0$. We define the shifted $N$-functions $\left\{\varphi_{a}\right\}_{a \geq 0}$ (cf. $[11,12,22]$ ) by

$$
\varphi_{a}(t):=\int_{0}^{t} \frac{\varphi^{\prime}(a+s) s}{a+s} \mathrm{~d} s, \quad t \geq 0 .
$$

Note that the family $\left\{\varphi_{a}\right\}_{a \geq 0}$ satisfies the $\Delta_{2}$-condition uniformly with respect to $a \geq 0$, i.e.,

$$
\sup _{a \geq 0} \sup _{t \geq 0} \frac{\varphi_{a}(2 t)}{\varphi_{a}(t)} \leq c 2^{\max \{2, p\}} .
$$

Definition 2.6 Let $\mathbf{S}: \mathbb{R}^{n \times n} \rightarrow \mathbb{R}_{\mathrm{sym}}^{n \times n}$ be a tensor field satisfying

$$
\mathbf{S} \in C^{0}\left(\mathbb{R}^{n \times n}, \mathbb{R}_{\mathrm{sym}}^{n \times n}\right) \cap C^{1}\left(\mathbb{R}^{n \times n} \backslash\{\mathbf{0}\}, \mathbb{R}_{\mathrm{sym}}^{n \times n}\right),
$$

$\mathbf{S}(\mathbf{P})=\mathbf{S}\left(\mathbf{P}^{\text {sym }}\right)$ whenever $P \in \mathbb{R}^{n \times n}$, and $\mathbf{S}(\mathbf{0})=\mathbf{0}$. We say that $\mathbf{S}$ has a $(p, \delta)$-structure if for some $p \in(1, \infty), \delta \in[0, \infty)$, and the $N$-function $\varphi=\varphi_{p, \delta}$ (cf. (2.15)) there exist constants $\gamma_{0}, \gamma_{1}>0$ such that the inequalities

$$
\begin{aligned}
\sum_{i, j, k, l=1}^{n} \partial_{k l} S_{i j}(\mathbf{P}) Q_{i j} Q_{k l} & \geq \gamma_{0} \varphi^{\prime \prime}\left(\left|\mathbf{P}^{\mathrm{sym}}\right|\right)\left|\mathbf{Q}^{\mathrm{sym}}\right|^{2}, \\
\left|\partial_{k l} S_{i j}(\mathbf{P})\right| & \leq \gamma_{1} \varphi^{\prime \prime}\left(\left|\mathbf{P}^{\mathrm{sym}}\right|\right)
\end{aligned}
$$

are satisfied for all $\mathbf{P}, \mathbf{Q} \in \mathbb{R}^{n \times n}$ with $\mathbf{P}^{\mathrm{sym}} \neq \mathbf{0}$ and all $i, j, k, l=1, \ldots, n$. The constants $\gamma_{0}, \gamma_{1}$, and $p$ are called the characteristics of $\mathbf{S}$.

Remark 2.7 An important example of a tensor field $\mathbf{S}$ having a $(p, \delta)$-structure is given by $\mathbf{S}(\mathbf{P})=\varphi^{\prime}\left(\left|\mathbf{P}^{\mathrm{sym}}\right|\right)\left|\mathbf{P}^{\mathrm{sym}}\right|^{-1} \mathbf{P}^{\mathrm{sym}}$. In this case, the characteristics of $\mathbf{S}$, namely $\gamma_{0}$ and $\gamma_{1}$, depend only on $p$ and are independent of $\delta \geq 0$.

Suppose that a tensor field $\mathbf{S}$ has a $(p, \delta)$-structure. Then we define its associated tensor field $\mathbf{F}: \mathbb{R}^{n \times n} \rightarrow \mathbb{R}_{\mathrm{sym}}^{n \times n}$ by

$$
\mathbf{F}(\mathbf{P}):=\left(\delta+\left|\mathbf{P}^{\mathrm{sym}}\right|\right)^{\frac{p-2}{2}} \mathbf{P}^{\mathrm{sym}} .
$$

The connection between $\mathbf{S}, \mathbf{F}$ and $\left\{\varphi_{a}\right\}_{a \geq 0}$ is best explained by the following proposition (cf. [11,22]).

Proposition 2.8 Let $\mathbf{S}$ have a $(p, \delta)$-structure, and let $\mathbf{F}$ be defined in (2.16). Then

$$
\begin{aligned}
(\mathbf{S}(\mathbf{P})-\mathbf{S}(\mathbf{Q})) \cdot(\mathbf{P}-\mathbf{Q}) & \sim|\mathbf{F}(\mathbf{P})-\mathbf{F}(\mathbf{Q})|^{2}, \\
& \sim \varphi_{\left|\mathbf{P}^{\mathrm{sym}}\right|}\left(\left|\mathbf{P}^{\mathrm{sym}}-\mathbf{Q}^{\mathrm{sym}}\right|\right), \\
& \sim \varphi^{\prime \prime}\left(\left|\mathbf{P}^{\mathrm{sym}}\right|+\left|\mathbf{P}^{\mathrm{sym}}-\mathbf{Q}^{\mathrm{sym}}\right|\right)\left|\mathbf{P}^{\mathrm{sym}}-\mathbf{Q}^{\mathrm{sym}}\right|^{2}, \\
|\mathbf{S}(\mathbf{P})-\mathbf{S}(\mathbf{Q})| & \sim \varphi_{\left|\mathbf{P}^{\mathrm{sym}}\right|}^{\prime}\left(\left|\mathbf{P}^{\mathrm{sym}}-\mathbf{Q}^{\mathrm{sym}}\right|\right),
\end{aligned}
$$

uniformly in $\mathbf{P}, \mathbf{Q} \in \mathbb{R}^{n \times n}$. Moreover, uniformly in $\mathbf{Q} \in \mathbb{R}^{n \times n}$,

$$
\mathbf{S}(\mathbf{Q}) \cdot \mathbf{Q} \sim|\mathbf{F}(\mathbf{Q})|^{2} \sim \varphi\left(\left|\mathbf{Q}^{\mathrm{sym}}\right|\right) .
$$

The constants depend only on the characteristics of $\mathbf{S}$. 
For a detailed discussion of the properties of $\mathbf{S}$ and $\mathbf{F}$ and their relation to Orlicz spaces and $N$-functions we refer the reader to [3,22]. In what follows, we shall work only with $\mathbf{S}(\mathbf{P})$ and $\mathbf{F}(\mathbf{P})$, where $\mathbf{P}$ is a symmetric tensor. Therefore, we can drop the superscript "sym" in the above formulas.

If $\mathbf{S}$ has a $(p, \delta)$-structure, from Proposition 2.8 we easily obtain the following equivalences:

$$
\begin{aligned}
\left|d_{h, k}^{ \pm} \mathbf{S}(\mathbf{D u})\right| & \sim\left(\delta+|\mathbf{D u}|+\left|\Delta_{h, k}^{ \pm} \mathbf{D u}\right|\right)^{p-2}\left|d_{h, k}^{ \pm} \mathbf{D u}\right| \\
& \sim \varphi^{\prime \prime}\left(|\mathbf{D u}|+\left|\Delta_{h, k}^{ \pm} \mathbf{D u}\right|\right)\left|d_{h, k}^{ \pm} \mathbf{D u}\right| \\
& \sim\left(\varphi^{\prime \prime}\left(|\mathbf{D u}|+\left|\Delta_{h, k}^{ \pm} \mathbf{D u}\right|\right)\right)^{\frac{1}{2}}\left|d_{h, k}^{ \pm} \mathbf{F}(\mathbf{D u})\right| \\
& \sim\left(\delta+|\mathbf{D u}|+\left|\Delta_{h, k}^{ \pm} \mathbf{D u}\right|\right)^{\frac{p-2}{2}}\left|d_{h, k}^{ \pm} \mathbf{F}(\mathbf{D u})\right|, \\
d_{h, k}^{ \pm} \mathbf{S}(\mathbf{D u}) \cdot d_{h, k}^{ \pm} \mathbf{D u} & \sim\left|d_{h, k}^{ \pm} \mathbf{F}(\mathbf{D u})\right|^{2} \\
& \sim\left(\delta+|\mathbf{D u}|+\left|\Delta_{h, k}^{ \pm} \mathbf{D u}\right|\right)^{p-2}\left|d_{h, k}^{ \pm} \mathbf{D u}\right|^{2} \\
& \sim \varphi^{\prime \prime}\left(|\mathbf{D u}|+\left|\Delta_{h, k}^{ \pm} \mathbf{D u}\right|\right)\left|d_{h, k}^{ \pm} \mathbf{D u}\right|^{2} .
\end{aligned}
$$

The equivalence constants depend here only on the characteristics of $\mathbf{S}$. All assertions from this section may be proved by easy manipulations of definitions, and we omit their proofs.

\section{Calderón-Zygmund estimates}

Our interest lies in estimates concerning Orlicz modulars. However, we are going to prove the results first in weighted $L^{p}$ spaces. The following known extrapolation principle (see [9, Theorem 4.15]) offers an elegant connection between the two settings.

Theorem 3.1 Let $p_{0} \in[1, \infty)$ and let $\mathcal{F}$ be a family of pairs of nonnegative measurable functions on $\mathbb{R}^{n}$. Suppose that there exists a nondecreasing function $C_{0}:[1, \infty) \rightarrow(0, \infty)$ such that the inequality

$$
\left(\int_{\mathbb{R}^{n}} f^{p_{0}}(\mathbf{x}) \omega(\mathbf{x}) \mathrm{d} \mathbf{x}\right)^{\frac{1}{p_{0}}} \leq C_{0}\left([\omega]_{A_{p_{0}}}\right)\left(\int_{\mathbb{R}^{n}} g^{p_{0}}(\mathbf{x}) \omega(\mathbf{x}) \mathrm{d} \mathbf{x}\right)^{\frac{1}{p_{0}}}
$$

holds for all $(f, g) \in \mathcal{F}$ and all weights $\omega \in A_{p_{0}}$. Then for every $N$-function $\psi$ satisfying $\Delta_{2}(\psi)<\infty$ and $\Delta_{2}\left(\psi^{*}\right)<\infty$ there exists a constant $C \in(0, \infty)$ such that the inequality

$$
\int_{\mathbb{R}^{n}} \psi(f(\mathbf{x})) \mathrm{d} \mathbf{x} \leq C \int_{\mathbb{R}^{n}} \psi(g(\mathbf{x})) \mathrm{d} \mathbf{x}
$$

holds for all $(f, g) \in \mathcal{F}$.

Remark 3.2 (i) The assumption on monotonicity of $C_{0}$ with respect to $[\omega]_{A_{p_{0}}}$ is necessary for the proof of Theorem 3.1. However, this is rarely explicitly mentioned in the literature.

(ii) Careful tracking of the constants in the proof of [9, Theorem 4.15] reveals that the constant $C$ in Theorem 3.1 depends on $C_{0}, \Delta_{2}(\psi)$ and $\Delta_{2}\left(\psi^{*}\right)$. To check this, one may use the inequality $\left[\omega_{1} \omega_{2}^{-1}\right]_{A_{2}} \leq\left[\omega_{1}\right]_{A_{1}}\left[\omega_{2}\right]_{A_{1}}$ (see [9, Proposition 3.5]) as well as the boundedness of the maximal operator in Orlicz spaces (see [17, Theorem 2.2]). The latter may be expressed by the inequality

$$
\int_{\mathbb{R}^{n}} \psi(|M f(\mathbf{x})|) \mathrm{d} \mathbf{x} \leq \Delta_{2}\left(\psi^{*}\right) \int_{\mathbb{R}^{n}} \psi\left(2 c^{2}|f(\mathbf{x})|\right) \mathrm{d} \mathbf{x},
$$


where $c$ is the weak $(1,1)$-constant of the maximal operator, which depends on $n$ only. In fact, one can show that

$$
C=\widetilde{c} C_{0}\left(2^{p_{0}} \rho_{\psi}(M)^{p_{0}-1} \rho_{\psi^{*}}(M)\right)^{\alpha} .
$$

In here, $\tilde{c}$ is a constant depending on $\Delta_{2}(\psi), \Delta_{2}\left(\psi^{*}\right)$, the exponent $\alpha$ depends on $\Delta_{2}(\psi)$, and $\rho_{\psi}(M)$ is the constant in the modular estimate of the maximal operator (it can be estimated by $\Delta_{2}\left(\psi^{*}\right) \Delta_{2}(\psi)^{\beta}$, where $\beta$ depends on $\left.n\right)$.

The auxiliary result below is a simple version of the Whitney covering lemma (cf. [2, p. 348]).

Proposition 3.3 Let $\Omega \in \mathbb{R}^{n}$ be a domain. Then there exists a sequence $\left\{Q_{j}\right\}_{j \in \mathbb{N}}$ of dyadic cubes satisfying:

(i) $\Omega=\bigcup_{j \in \mathbb{N}} \bar{Q}_{j}$,

(ii) $Q_{j} \cap Q_{k} \neq \varnothing \Leftrightarrow j=k$,

(iii) $\operatorname{dist}\left(Q_{j}, \partial \Omega\right)>4$ diam $Q_{j}$ for all $j \in \mathbb{N}$.

Proof Let $\left\{\mathbf{x}^{k}\right\}_{k \in \mathbb{N}}$ be a dense sequence of points in $\Omega$. Define $Q_{1}$ as the largest ${ }^{1}$ dyadic cube $Q$ such that $\mathbf{x}^{1} \in \bar{Q}$ and dist $(Q, \partial \Omega)>4$ diam $Q$. Next, suppose that $Q_{1}, \ldots, Q_{j-1}$ are defined and let $k \in \mathbb{N}$ be the smallest index such that $\mathbf{x}^{k} \notin \bigcup_{i=1}^{j-1} Q_{i}$. Then define $Q_{j}$ as the largest dyadic cube $Q$ such that $\mathbf{x}^{k} \in \bar{Q}$ and dist $(Q, \partial \Omega)>4$ diam $Q$. The sequence $\left\{Q_{j}\right\}_{j \in \mathbb{N}}$ obviously satisfies (ii) and (iii). To verify (i), let $\mathbf{x} \in \Omega$ be arbitrary. There exists a dyadic cube $Q$ such that $\mathbf{x} \in \bar{Q}$ and dist $(Q, \partial \Omega)>4$ diam $Q$. By density, $\mathbf{x}^{k} \in Q$ for some $k \in \mathbb{N}$. Then necessarily $\bar{Q} \subseteq \bar{Q}_{j}$ for some $j \in \mathbb{N}$, hence $\mathbf{x} \in \bigcup_{j \in \mathbb{N}} \bar{Q}_{j}$.

The next theorem justifies the definition of Calderón-Zygmund operators with standard kernels by the Cauchy principal value of the integral (2.11) and shows their boundedness in $L_{\omega}^{p}(\Omega)$ and $L^{\psi}(\Omega)$. Although the result is well-known, in standard literature it appears only in the setting $\Omega=\mathbb{R}^{n}$. We prove it below for any domain $\Omega \subset \mathbb{R}^{n}$.

Theorem 3.4 Let $\Omega \subset \mathbb{R}^{n}$ be a domain. Let $K \in \mathrm{SK}\left(\Omega, \kappa_{1}\right) \cap \mathrm{CZ}\left(\Omega, \kappa_{2}\right)$ for some $\kappa_{1}, \kappa_{2} \in$ $(0, \infty)$. Then the following assertions hold true:

(i) $L e t T_{\varepsilon}$ be the operators generated by the truncated kernels $K_{\varepsilon}$. Then for any $f \in C_{0}^{\infty}(\Omega)$ the expressions

$$
\begin{aligned}
T f(\mathbf{x}) & :=\lim _{\varepsilon \rightarrow 0} T_{\varepsilon} f(\mathbf{x}), \\
T^{(*)} f(\mathbf{x}) & :=\sup _{\varepsilon>0}\left|T_{\varepsilon} f(\mathbf{x})\right|
\end{aligned}
$$

are defined for a.e. $\mathbf{x} \in \Omega$.

(ii) For every $p \in(1, \infty)$ there exists a nondecreasing function $C_{p}:[1, \infty) \rightarrow(0, \infty)$ such that the operator $T$ defined by (3.1) admits, for each $\omega \in A_{p}$, a bounded extension to $L_{\omega}^{p}(\Omega)$ satisfying

$$
\left(\int_{\Omega}|T f(\mathbf{x})|^{p} \omega(\mathbf{x}) \mathrm{d} \mathbf{x}\right)^{\frac{1}{p}} \leq C_{p}\left([\omega]_{A_{p}}\right)\left(\kappa_{1}+\kappa_{2}\right)\left(\int_{\Omega}|f(\mathbf{x})|^{p} \omega(\mathbf{x}) \mathrm{d} \mathbf{x}\right)^{\frac{1}{p}}
$$

for all $f \in L_{\omega}^{p}(\Omega)$. An analogous assertion holds for the operator $T^{(*)}$.

${ }^{1}$ Such cube exists since if a point is contained in two dyadic cubes, then one of the cubes is a subset of the other one. 
(iii) For every $N$-function $\psi$ with $\Delta_{2}(\psi), \Delta_{2}\left(\psi^{*}\right)<\infty$ there exists a positive constant $C_{\psi}$ such that the operator $T$ defined by (3.1) admits a bounded extension to $L^{\psi}(\Omega)$ satisfying

$$
\int_{\Omega} \psi(|T f(\mathbf{x})|) \mathrm{d} \mathbf{x} \leq C_{\psi} \int_{\Omega} \psi\left(\left(\kappa_{1}+\kappa_{2}\right)|f(\mathbf{x})|\right) \mathrm{d} \mathbf{x}
$$

for all $f \in L^{\psi}(\Omega)$. An analogous assertion holds for the operator $T^{(*)}$.

Remark 3.5 In the above theorem, $C_{p}$ and $C_{\psi}$ are independent of the domain $\Omega$ in the following sense. Let $\Omega, \Omega^{\prime} \subset \mathbb{R}^{n}$ be domains, let $K, K^{\prime}$ be kernels such that $K \in$ $\operatorname{SK}\left(\Omega, \kappa_{1}\right) \cap \operatorname{CZ}\left(\Omega, \kappa_{2}\right), K^{\prime} \in \operatorname{SK}\left(\Omega^{\prime}, \kappa_{1}\right) \cap \operatorname{CZ}\left(\Omega^{\prime}, \kappa_{2}\right)$ with the same $\kappa_{1}, \kappa_{2} \in(0, \infty)$, and let $T, T^{\prime}$ be the corresponding operators. Then, for each $p \in(1, \infty)$, inequality (3.2) holds with the same $C_{p}$ when $\Omega$ is replaced by $\Omega^{\prime}$ and $T$ by $T^{\prime}$. An analogous assertion holds regarding (3.3).

Proof of Theorem 3.4 We are going to prove the theorem only for the operator $T$. The proof for $T^{(*)}$ follows the same reasoning. At first, let us assume that

$$
K \in \mathrm{SK}\left(\widetilde{\Omega}, \kappa_{1}\right) \cap \mathrm{CZ}\left(\widetilde{\Omega}, \kappa_{2}\right),
$$

where

$$
\widetilde{\Omega}:=\left\{\mathbf{x} \in \mathbb{R}^{n} \mid \operatorname{dist}(\mathbf{x}, \Omega) \leq 4 \operatorname{diam} \Omega\right\} .
$$

Let $\eta \in C_{0}^{\infty}\left(\mathbb{R}^{n}\right)$ satisfy $0 \leq \eta \leq 1,\left.\eta\right|_{\Omega} \equiv 1, \eta(\mathbf{x})=0$ for all $\mathbf{x} \in \mathbb{R}^{n}$ such that $\operatorname{dist}(\mathbf{x}, \Omega)>\operatorname{diam} \Omega$, and $\|\nabla \eta\|_{\infty} \leq 2(\operatorname{diam} \Omega)^{-1}$. For $\mathbf{x}, \mathbf{y} \in \mathbb{R}^{n}$ define

$$
\widehat{K}(\mathbf{x}, \mathbf{y}):=K(\mathbf{x}, \mathbf{y}) \eta(\mathbf{x}), \quad \text { and } \quad \widetilde{K}(\mathbf{x}, \mathbf{y}):=K(\mathbf{x}, \mathbf{y}) \eta(\mathbf{x}) \eta(\mathbf{y}) .
$$

Then the kernel

$$
\widehat{N}(\mathbf{x}, \mathbf{y}):=\widehat{K}(\mathbf{x}, \mathbf{x}-\mathbf{y})=K(\mathbf{x}, \mathbf{x}-\mathbf{y}) \eta(\mathbf{x})=N(\mathbf{x}, \mathbf{y}) \eta(\mathbf{x})
$$

satisfies the conditions (2.12) and (2.13). Moreover, it satisfies (2.14) globally, i.e., with $E=\mathbb{R}^{n}$. Thus, $\widehat{K} \in \mathrm{CZ}\left(\mathbb{R}^{n}, \kappa_{2}\right)$. Therefore, by [7, Theorem 2],

$$
\widehat{T} g(\mathbf{x}):=\lim _{\varepsilon \rightarrow 0} \int_{\mathbb{R}^{n}} g(\mathbf{y}) \widehat{K}_{\varepsilon}(\mathbf{x}, \mathbf{y}) \mathrm{d} \mathbf{y}=\lim _{\varepsilon \rightarrow 0} \widehat{T}_{\varepsilon} g(\mathbf{x})
$$

is defined for a.e. $\mathbf{x} \in \mathbb{R}^{n}$ and all $g \in C_{0}^{\infty}\left(\mathbb{R}^{n}\right)$ and it admits a bounded extension from $L^{2}\left(\mathbb{R}^{n}\right)$ to $L^{2}\left(\mathbb{R}^{n}\right)$. It follows from the proofs and comments in [7], in particular p. 295 and a remark on p. 306, that the corresponding operator norm satisfies $\|\widehat{T}\|_{L^{2} \rightarrow L^{2}} \leq c \kappa_{2}$, where $c>0$ is a fixed constant depending only on the dimension $n$. Since $\widetilde{T}_{\varepsilon} g(\mathbf{x})=\widehat{T}_{\varepsilon}(g \eta)(\mathbf{x})$ holds for all $\varepsilon>0, g \in C_{0}^{\infty}\left(\mathbb{R}^{n}\right)$ and a.e. $\mathbf{x} \in \mathbb{R}^{n}$, we may draw the same conclusions about the operator

$$
\widetilde{T} g(\mathbf{x}):=\lim _{\varepsilon \rightarrow 0} \int_{\mathbb{R}^{n}} g(\mathbf{y}) \widetilde{K}_{\varepsilon}(\mathbf{x}, \mathbf{y}) \mathrm{d} \mathbf{y}=\lim _{\varepsilon \rightarrow 0} \widetilde{T}_{\varepsilon} g(\mathbf{x}) .
$$

In particular, we get $\widetilde{T} g(\mathbf{x})=\widehat{T}(g \eta)(\mathbf{x})$, and thus

$$
\|\widetilde{T}\|_{L^{2} \rightarrow L^{2}} \leq\|\widehat{T}\|_{L^{2} \rightarrow L^{2}} \leq c \kappa_{2} \text {. }
$$

Let us next show that $\widetilde{K}$ is a standard kernel with respect to $\mathbb{R}^{n}$. Suppose that $\mathbf{x}, \mathbf{y}, \mathbf{z} \in \mathbb{R}^{n}$ satisfy (2.7). Then

$$
|\mathbf{x}-\mathbf{y}| \leq 2|\mathbf{z}-\mathbf{y}| \leq 3|\mathbf{x}-\mathbf{y}|
$$


We will distinguish two cases. At first, assume that

$$
\mathbf{x}, \mathbf{z} \in \widetilde{\Omega} \text { and } \operatorname{dist}(\mathbf{y}, \Omega) \leq \operatorname{diam} \Omega .
$$

Then obviously $y \in \widetilde{\Omega}$, and since $K \in \operatorname{SK}\left(\widetilde{\Omega}, \kappa_{1}\right)$, we have

$$
\begin{aligned}
|\widetilde{K}(\mathbf{x}, \mathbf{y})| & =|K(\mathbf{x}, \mathbf{y}) \eta(\mathbf{x}) \eta(\mathbf{y})| \leq \frac{\kappa_{1}}{|\mathbf{x}-\mathbf{y}|^{n}}, \\
|\widetilde{K}(\mathbf{x}, \mathbf{y})-\widetilde{K}(\mathbf{z}, \mathbf{y})| & =|K(\mathbf{x}, \mathbf{y}) \eta(\mathbf{x}) \eta(\mathbf{y})-K(\mathbf{z}, \mathbf{y}) \eta(\mathbf{z}) \eta(\mathbf{y})| \\
& \leq|K(\mathbf{x}, \mathbf{y})-K(\mathbf{z}, \mathbf{y})||\eta(\mathbf{x}) \eta(\mathbf{y})|+|K(\mathbf{z}, \mathbf{y})||\eta(\mathbf{x})-\eta(\mathbf{z})||\eta(\mathbf{y})| \\
& \leq\left(1+3 \cdot 2^{n+2}\right) \kappa_{1} \frac{|\mathbf{x}-\mathbf{z}|}{|\mathbf{x}-\mathbf{y}|^{n+1}}, \\
|\widetilde{K}(\mathbf{y}, \mathbf{x})-\widetilde{K}(\mathbf{y}, \mathbf{z})| & \leq|K(\mathbf{y}, \mathbf{x})-K(\mathbf{y}, \mathbf{z})||\eta(\mathbf{x}) \eta(\mathbf{y})|+|K(\mathbf{y}, \mathbf{z})||\eta(\mathbf{x})-\eta(\mathbf{z}) \| \eta(\mathbf{y})| \\
& \leq\left(1+3 \cdot 2^{n+2}\right) \kappa_{1} \frac{|\mathbf{x}-\mathbf{z}|}{|\mathbf{x}-\mathbf{y}|^{n+1}} .
\end{aligned}
$$

In the second and third condition we used the estimate

$$
\frac{|\mathbf{x}-\mathbf{z}|}{|\mathbf{z}-\mathbf{y}|^{n}}=\frac{|\mathbf{x}-\mathbf{z}||\mathbf{x}-\mathbf{y}|}{|\mathbf{z}-\mathbf{y}|^{n}|\mathbf{x}-\mathbf{y}|} \leq \frac{3 \cdot 2^{n+1} \operatorname{diam} \Omega|\mathbf{x}-\mathbf{z}|}{|\mathbf{x}-\mathbf{y}|^{n+1}},
$$

which holds due to (3.6) and (3.7), and the property $\|\nabla \eta\|_{\infty} \leq 2(\operatorname{diam} \Omega)^{-1}$.

Now suppose that (2.7) holds but (3.7) does not. Then one of the following situations occurs:

(i) $\operatorname{dist}(\mathbf{y}, \Omega)>\operatorname{diam} \Omega$, in which case $\mathbf{y} \notin \operatorname{supp} \eta$.

(ii) $\operatorname{dist}(\mathbf{y}, \Omega) \leq \operatorname{diam} \Omega$ and $\operatorname{dist}(\mathbf{x}, \Omega)>4 \operatorname{diam} \Omega$. Then $\mathbf{x} \notin \operatorname{supp} \eta$ and, moreover, we get

$$
\begin{aligned}
\operatorname{dist}(\mathbf{x}, \Omega) & \leq \operatorname{dist}(\mathbf{z}, \Omega)+|\mathbf{x}-\mathbf{z}| \leq \operatorname{dist}(\mathbf{z}, \Omega)+\frac{1}{2}|\mathbf{x}-\mathbf{y}| \\
& \leq \operatorname{dist}(\mathbf{z}, \Omega)+\frac{1}{2}(\operatorname{dist}(\mathbf{x}, \Omega)+\operatorname{dist}(\mathbf{y}, \Omega)+\operatorname{diam} \Omega) \\
& \leq \operatorname{dist}(\mathbf{z}, \Omega)+\frac{1}{2} \operatorname{dist}(\mathbf{x}, \Omega)+\operatorname{diam} \Omega
\end{aligned}
$$

Hence,

$$
\operatorname{dist}(\mathbf{z}, \Omega) \geq \frac{1}{2} \operatorname{dist}(\mathbf{x}, \Omega)-\operatorname{diam} \Omega>\operatorname{diam} \Omega,
$$

which shows that $\mathbf{z} \notin$ supp $\eta$.

(iii) $\operatorname{dist}(\mathbf{y}, \Omega) \leq \operatorname{diam} \Omega$ and $\operatorname{dist}(\mathbf{z}, \Omega)>4 \operatorname{diam} \Omega$. Then $\mathbf{z} \notin$ supp $\eta$ and, moreover, we get

$$
\begin{aligned}
\operatorname{dist}(\mathbf{z}, \Omega) & \leq \operatorname{dist}(\mathbf{x}, \Omega)+|\mathbf{x}-\mathbf{z}| \leq \operatorname{dist}(\mathbf{x}, \Omega)+\frac{1}{2}|\mathbf{x}-\mathbf{y}| \\
& \leq \operatorname{dist}(\mathbf{x}, \Omega)+\frac{1}{2}(\operatorname{dist}(\mathbf{x}, \Omega)+\operatorname{dist}(\mathbf{y}, \Omega)+\operatorname{diam} \Omega) \\
& \leq \frac{3}{2} \operatorname{dist}(\mathbf{x}, \Omega)+\operatorname{diam} \Omega .
\end{aligned}
$$

Hence,

$$
\operatorname{dist}(\mathbf{x}, \Omega) \geq 2 \operatorname{diam} \Omega,
$$

which shows that $\mathbf{x} \notin \operatorname{supp} \eta$. 
In each of the three cases we get

$$
\widetilde{K}(\mathbf{x}, \mathbf{y})=\widetilde{K}(\mathbf{z}, \mathbf{y})=\widetilde{K}(\mathbf{y}, \mathbf{x})=\widetilde{K}(\mathbf{y}, \mathbf{z})=0,
$$

hence the conditions (2.8)-(2.10) are satisfied trivially. Altogether, we have now verified that $\widetilde{K} \in \mathrm{SK}\left(\mathbb{R}^{n}, \kappa_{0}\right)$ with the constant $\kappa_{0}=:\left(1+3 \cdot 2^{n+2}\right) \kappa_{1}$. Thanks to this and the estimate (3.5), $[18$, Theorem 7.4.6] yields that for every $p \in(1, \infty)$ there exists a nondecreasing function $C_{p}:[1, \infty) \rightarrow(0, \infty)$ such that

$$
\left(\int_{\mathbb{R}^{n}}|\tilde{T} f(\mathbf{x})|^{p} \omega(\mathbf{x}) \mathrm{d} \mathbf{x}\right)^{\frac{1}{p}} \leq C_{p}\left([\omega]_{A_{p}}\right)\left(\kappa_{1}+\kappa_{2}\right)\left(\int_{\mathbb{R}^{n}}|f(\mathbf{x})|^{p} \omega(\mathbf{x}) \mathrm{d} \mathbf{x}\right)^{\frac{1}{p}}
$$

holds for all $f \in C_{0}^{\infty}\left(\mathbb{R}^{n}\right)$ and all $\omega \in A_{p}$. It has to be noted that the desired monotone dependence of $C_{p}$ on $[\omega]_{A_{p}}$ is not mentioned in [18] but it can be verified through a careful inspection of the proofs leading to the result in there. Notice also that $C_{p}$ is independent of $\kappa_{1}, \kappa_{2}$ and $\Omega$.

In the next step, let $\psi$ be an $N$-function with $\Delta_{2}(\psi)<\infty$ and $\Delta_{2}\left(\psi^{*}\right)<\infty$. Due to (3.8) Theorem 3.1 provides the existence of a positive constant $C_{\psi}$ such that

$$
\int_{\mathbb{R}^{n}} \psi(|\widetilde{T} f(\mathbf{x})|) \mathrm{d} \mathbf{x} \leq C_{\psi} \int_{\mathbb{R}^{n}} \psi\left(\left(\kappa_{1}+\kappa_{2}\right)|f(\mathbf{x})|\right) \mathrm{d} \mathbf{x}
$$

for all $f \in C_{0}^{\infty}\left(\mathbb{R}^{n}\right)$. To obtain this estimate we have performed the extrapolation with respect to the family

$$
\mathcal{F}:=\left\{\left(\widetilde{T} f,\left(\kappa_{1}+\kappa_{2}\right) f\right) \mid f \in C_{0}^{\infty}\left(\mathbb{R}^{n}\right)\right\} .
$$

The obtained constant $C_{\psi}$ is independent of $\kappa_{1}, \kappa_{2}$ and $\Omega$. For any $f \in C_{0}^{\infty}(\Omega)$ and $\mathbf{x} \in \Omega$ we have

$$
\widetilde{T} f(\mathbf{x})=\eta(\mathbf{x}) T f(\mathbf{x})=T f(\mathbf{x}) .
$$

Hence, (3.9) and (3.8) yield (3.2) and (3.3), respectively, for $f \in C_{0}^{\infty}(\Omega)$. Since $C_{0}^{\infty}(\Omega)$ is dense in $L_{\omega}^{p}(\Omega)$ as well as in $L^{\psi}(\Omega)$, the operator $T$ admits corresponding bounded extensions such that (3.2) and (3.3) hold for any $f \in L_{\omega}^{p}(\Omega)$ and $f \in L^{\psi}(\Omega)$, respectively.

So far, we have proved the theorem under the stronger assumption (3.4). To prove it in full generality, let $\Omega \subset \mathbb{R}^{n}$ be an arbitrary domain and $K \in \operatorname{SK}\left(\Omega, \kappa_{1}\right) \cap \operatorname{CZ}\left(\Omega, \kappa_{2}\right)$. By Proposition 3.3 there exists a sequence $\left\{Q_{j}\right\}_{j \in \mathbb{N}}$ of pairwise disjoint dyadic cubes such that $\Omega=\bigcup_{j \in \mathbb{N}} \bar{Q}_{j}$ and

$$
\widetilde{Q}_{j}:=\left\{\mathbf{x} \in \mathbb{R}^{n} \mid \operatorname{dist}\left(\mathbf{x}, Q_{j}\right) \leq 4 \operatorname{diam} Q_{j}\right\} \subset \Omega
$$

for all $j \in \mathbb{N}$. Hence, for any $j \in \mathbb{N}$, we have $K \in \mathrm{SK}\left(\widetilde{Q}_{j}, \kappa_{1}\right) \cap \mathrm{CZ}\left(\widetilde{Q}_{j}, \kappa_{2}\right)$, and using the first part of the proof we get

$$
\begin{aligned}
\int_{\Omega}|T f(\mathbf{x})|^{p} \omega(\mathbf{x}) \mathrm{d} \mathbf{x} & =\sum_{j \in \mathbb{N}} \int_{Q_{j}}|T f(\mathbf{x})|^{p} \omega(\mathbf{x}) \mathrm{d} \mathbf{x} \\
& \leq C_{p}^{p}\left([\omega]_{A_{p}}\right)\left(\kappa_{1}+\kappa_{2}\right)^{p} \sum_{j \in \mathbb{N}} \int_{Q_{j}}|f(\mathbf{x})|^{p} \omega(\mathbf{x}) \mathrm{d} \mathbf{x} \\
& =C_{p}^{p}\left([\omega]_{A_{p}}\right)\left(\kappa_{1}+\kappa_{2}\right)^{p} \int_{\Omega}|f(\mathbf{x})|^{p} \omega(\mathbf{x}) \mathrm{d} \mathbf{x}
\end{aligned}
$$

for all $\omega \in A_{p}$ and $f \in L_{\omega}^{p}(\Omega)$. In here, it is important that $C_{p}^{p}\left([\omega]_{A_{p}}\right)$ does not depend on $Q_{j}$. Similarly, we obtain (3.3) for any $f \in L^{\psi}(\Omega)$. Note also that Remark 3.5 is justified. 


\section{Divergence equation}

We proceed with proving the new estimate concerning the Bogovski solution of the divergence equation. We prove the result in the weighted- $L^{p}$ setting. The variant for Orlicz modulars will be then obtained as a corollary by extrapolation.

Theorem 4.1 Let $Q \subset \mathbb{R}^{n}$ be an open cube. There exists a linear operator $\mathcal{B}: C_{0,0}^{\infty}(Q) \rightarrow$ $W_{0}^{1, \infty}(Q)$ with the following properties:

(i) For every $f \in C_{0,0}^{\infty}(Q)$, the equation

$$
\operatorname{div}(\mathcal{B} f)=f
$$

is satisfied in $Q$.

(ii) For every $p \in(1, \infty)$ there exists a nondecreasing function $C_{p}:[1, \infty) \rightarrow(0, \infty)$ such that

$$
\left(\int_{Q}|\nabla(\mathcal{B} f)(\mathbf{x})|^{p} \omega(\mathbf{x}) \mathrm{d} \mathbf{x}\right)^{\frac{1}{p}} \leq C_{p}\left([\omega]_{A_{p}}\right)\left(\int_{Q}|f(\mathbf{x})|^{p} \omega(\mathbf{x}) \mathrm{d} \mathbf{x}\right)^{\frac{1}{p}} .
$$

holds for all $\omega \in A_{p}$ and all $f \in C_{0,0}^{\infty}(Q)$. The function $C_{p}$ is independent of $Q$.

(iii) For every $p \in(1, \infty)$ there exists a nondecreasing function $\widetilde{C}_{p}:[1, \infty) \rightarrow(0, \infty)$ such that

$$
\begin{aligned}
& \left(\int_{Q}\left|d_{h, k}^{ \pm} \nabla(\mathcal{B} f)(\mathbf{x})\right|^{p} \omega(\mathbf{x}) \mathrm{d} \mathbf{x}\right)^{\frac{1}{p}} \\
& \quad \leq \widetilde{C}_{p}\left([\omega]_{A_{p}}\right)\left(\int_{Q}\left(\left|d_{h, k}^{+} f(\mathbf{x})\right|+\left|d_{h, k}^{-} f(\mathbf{x})\right|+\frac{|f(\mathbf{x})|}{\ell(Q)}\right)^{p} \omega(\mathbf{x}) \mathrm{d} \mathbf{x}\right)^{\frac{1}{p}}
\end{aligned}
$$

holds for all $h>0, \omega \in A_{p}$ and $f \in C_{0,0}^{\infty}(Q)$. The function $\widetilde{C}_{p}$ is independent of $Q$.

(iv) For every $p \in(1, \infty)$ and $\omega \in A_{p}$ there exists a continuous extension of $\mathcal{B}$ to $L_{\omega, 0}^{p}(Q)$ such that (4.1), (4.2) and (4.3) hold for all $f \in L_{\omega, 0}^{p}(Q)$. In here, $f$ and $\mathcal{B} f$ are extended by zero outside $Q$ so that their difference quotients are defined a.e. in $\mathbb{R}^{n}$.

Corollary 4.2 Let $Q \subset \mathbb{R}^{n}$ be an open cube. Let $\psi$ be an $N$-function with $\Delta_{2}(\psi)<\infty$ and $\Delta_{2}\left(\psi^{*}\right)<\infty$. Then there exists a linear operator $\mathcal{B}: L_{0}^{\psi}(Q) \rightarrow W_{0}^{1, \psi}(Q)$ with the following properties:

(i) Equation (4.1) is satisfied a.e. in $Q$ for all $f \in L_{0}^{\psi}(Q)$.

(ii) There exists a positive constant $C_{\psi}$ such that

$$
\int_{Q} \psi(|\nabla(\mathcal{B} f)(\mathbf{x})|) \mathrm{d} \mathbf{x} \leq C_{\psi} \int_{Q} \psi(|f(\mathbf{x})|) \mathrm{d} \mathbf{x}
$$

holds for all $f \in L_{0}^{\psi}(Q)$. The constant $C_{\psi}$ is independent of $Q$.

(iii) There exists a positive constant $\widetilde{C}_{\psi}$ such that

$$
\int_{Q} \psi\left(\left|d_{h, k}^{ \pm} \nabla(\mathcal{B} f)(\mathbf{x})\right|\right) \mathrm{d} \mathbf{x} \leq \widetilde{C}_{\psi} \int_{Q} \psi\left(\left|d_{h, k}^{+} f(\mathbf{x})\right|+\left|d_{h, k}^{-} f(\mathbf{x})\right|+\frac{|f(\mathbf{x})|}{\ell(Q)}\right) \mathrm{d} \mathbf{x}
$$

holds for all $h>0$ and all $f \in L_{0}^{\psi}(Q)$. In here, $f$ and $\mathcal{B} f$ are extended by zero outside $Q$ so that their difference quotients are defined a.e. in $\mathbb{R}^{n}$. The constant $\widetilde{C}_{\psi}$ is independent of $Q$. 
Before we prove Theorem 4.1, let us show the following simple technical proposition.

Lemma 4.3 Let $\varrho \in C^{\infty}\left(\mathbb{R}^{n}, \mathbb{R}\right)$ and $\mathbf{a}, \mathbf{b}, \mathbf{w} \in \mathbb{R}^{n}$. Then

$$
|\varrho(\mathbf{a})-\varrho(\mathbf{a}+\mathbf{w})-\varrho(\mathbf{b})+\varrho(\mathbf{b}+\mathbf{w})| \leq\left\|\nabla^{2} \varrho\right\|_{\infty}|\mathbf{w}||\mathbf{a}-\mathbf{b}| .
$$

Proof We have

$$
\begin{aligned}
|\varrho(\mathbf{a})-\varrho(\mathbf{a}+\mathbf{w})-\varrho(\mathbf{b})+\varrho(\mathbf{b}+\mathbf{w})| & \leq\|\nabla \varrho-\nabla \varrho(\cdot+\mathbf{w})\|_{\infty}|\mathbf{a}-\mathbf{b}| \\
& \leq\left\|\nabla^{2} \varrho\right\|_{\infty}|\mathbf{w}| \mathbf{a}-\mathbf{b} \mid .
\end{aligned}
$$

Proof of Theorem 4.1 At first suppose that $Q=\left(-\frac{1}{2}, \frac{1}{2}\right)^{n}$. Choose a fixed function $\varrho \in$ $C_{0}^{\infty}\left(\mathbb{R}^{n}\right)$ such that supp $\varrho \subset\left(-\frac{1}{4}, \frac{1}{4}\right)^{n}$ and $\int_{Q} \varrho(\mathbf{x}) \mathrm{d} \mathbf{x}=1$. For any $f \in C_{0,0}^{\infty}(Q)$ define

$$
\mathcal{B} f(\mathbf{x}):=\int_{Q} f(\mathbf{y})\left(\frac{\mathbf{x}-\mathbf{y}}{|\mathbf{x}-\mathbf{y}|^{n}} \int_{|\mathbf{x}-\mathbf{y}|}^{\infty} \varrho\left(\mathbf{y}+\xi \frac{\mathbf{x}-\mathbf{y}}{|\mathbf{x}-\mathbf{y}|}\right) \xi^{n-1} \mathrm{~d} \xi\right) \mathrm{d} \mathbf{y} .
$$

From [16, Chapter III, Lemma 3.1] it follows that $\mathcal{B} f \in W_{0}^{1, \infty}(Q)$ and it satisfies (4.1) in $Q$.

From now on, we will use the notation

$$
\text { p.v. } \int_{E} g(\mathbf{y}) K(\mathbf{x}, \mathbf{y}) \mathrm{d} \mathbf{y}:=\lim _{\varepsilon \rightarrow 0} \int_{E \cap\{|\mathbf{x}-\mathbf{y}|>\varepsilon\}} g(\mathbf{y}) K(\mathbf{x}, \mathbf{y}) \mathrm{d} \mathbf{y}
$$

for functions $g \in C_{0}^{\infty}\left(\mathbb{R}^{n}\right)$ and kernels $K$ whenever the limit on the right-hand side exists.

Part (ii) is proved in $[13,23]$ without the monotone dependence of $C_{p}$ on $[\omega]_{A_{p}}$. Note that this dependence of the constant is mentioned in [24]. For the sake of the self-consistency of this paper we sketch its proof here. The technique used here is the same as in part (iii), where full details will be given. Let $i, j \in\{1, \ldots, n\}$. Since $Q$ is convex and it contains supp $\varrho$, by [16, p. 119] we have

$$
\begin{aligned}
\partial_{j} \mathcal{B} f_{i}(\mathbf{x})=\text { p.v. } \int_{Q} f(\mathbf{y}) J_{i j}(\mathbf{x}, \mathbf{y}) \mathrm{d} \mathbf{y}+\int_{Q} f(\mathbf{y}) U_{i j}(\mathbf{x}, \mathbf{y}) \mathrm{d} \mathbf{y} \\
+f(\mathbf{x}) \int_{Q} \frac{\left(x_{i}-y_{i}\right)\left(x_{j}-y_{j}\right)}{|\mathbf{x}-\mathbf{y}|^{2}} \varrho(\mathbf{y}) \mathrm{d} \mathbf{y}
\end{aligned}
$$

for any $f \in C_{0,0}^{\infty}(Q)$. In here,

$$
\begin{aligned}
J_{i j}(\mathbf{x}, \mathbf{y}):= & \frac{\delta_{i j}}{|\mathbf{x}-\mathbf{y}|^{n}} \int_{0}^{\infty} \varrho\left(\mathbf{x}+\xi \frac{\mathbf{x}-\mathbf{y}}{|\mathbf{x}-\mathbf{y}|}\right) \xi^{n-1} \mathrm{~d} \xi \\
& +\frac{x_{i}-y_{i}}{|\mathbf{x}-\mathbf{y}|^{n+1}} \int_{0}^{\infty} \partial_{j} \varrho\left(\mathbf{x}+\xi \frac{\mathbf{x}-\mathbf{y}}{|\mathbf{x}-\mathbf{y}|}\right) \xi^{n} \mathrm{~d} \xi
\end{aligned}
$$

and $U_{i j}$ is a kernel such that $\left|U_{i j}(\mathbf{x}, \mathbf{y})\right| \leq c\|\varrho\|_{1, \infty}|\mathbf{x}-\mathbf{y}|^{1-n}$, where $c$ is a positive constant. From [10, Lemma 6.1] we get that $J_{i j} \in \mathrm{SK}(Q, \kappa) \cap \mathrm{CZ}(Q, \kappa)$, where $\kappa$ depends only on $\|\varrho\|_{1, \infty}$ and $n$. By Theorem 3.4 there exists a positive nondecreasing function $c_{p}$ such that

$$
\left(\int_{Q} \mid \text { p.v. }\left.\int_{Q} f(\mathbf{y}) J_{i j}(\mathbf{x}, \mathbf{y}) \mathrm{d} \mathbf{y}\right|^{p} \omega(\mathbf{x}) \mathrm{d} \mathbf{x}\right)^{\frac{1}{p}} \leq c_{p}\left([\omega]_{A_{p}}\right)\left(\int_{Q}|f(\mathbf{x})|^{p} \omega(\mathbf{x}) \mathrm{d} \mathbf{x}\right)^{\frac{1}{p}}
$$


for all $f \in C_{0,0}^{\infty}(Q)$. Furthermore, we have (see [10, p. 218])

$$
\int_{Q} f(\mathbf{y}) U_{i j}(\mathbf{x}, \mathbf{y}) \mathrm{d} \mathbf{y} \leq 2^{n} c\|\varrho\|_{1, \infty} M f(\mathbf{x})
$$

for $f \in C_{0,0}^{\infty}(Q)$ and $\mathbf{x} \in Q$. Hence, by [18, Theorem 7.1.9(b)] there exists a positive constant $C$ (possibly depending on $p, n$ ) such that

$$
\left(\int_{Q}\left|\int_{Q} f(\mathbf{y}) U_{i j}(\mathbf{x}, \mathbf{y}) \mathrm{d} \mathbf{y}\right|^{p} \omega(\mathbf{x}) \mathrm{d} \mathbf{x}\right)^{\frac{1}{p}} \leq C[\omega]_{A_{p}}^{\frac{1}{p-1}}\|\varrho\|_{1, \infty}\left(\int_{Q}|f(\mathbf{x})|^{p} \omega(\mathbf{x}) \mathrm{d} \mathbf{x}\right)^{\frac{1}{p}}
$$

for all $f \in C_{0,0}^{\infty}(Q)$. Obviously, we also have

$$
\left(\int_{Q}\left|f(\mathbf{x}) \int_{Q} \frac{\left(x_{i}-y_{i}\right)\left(x_{j}-y_{j}\right)}{|\mathbf{x}-\mathbf{y}|^{2}} \varrho(\mathbf{y}) \mathrm{d} \mathbf{y}\right|^{p} \omega(\mathbf{x}) \mathrm{d} \mathbf{x}\right)^{\frac{1}{p}} \leq\|\varrho\|_{1}\left(\int_{Q}|f(\mathbf{x})|^{p} \omega(\mathbf{x}) \mathrm{d} \mathbf{x}\right)^{\frac{1}{p}} .
$$

Therefore, by combining (4.6), (4.7) and (4.8), we have shown that there exists a positive nondecreasing function $C_{p}$ such that (4.2) holds for all $f \in C_{0,0}^{\infty}(Q)$. Recall however that so far we have assumed $Q=\left(-\frac{1}{2}, \frac{1}{2}\right)^{n}$. The result for a general cube will be obtained by rescaling at the end of the proof of part (iii).

We continue with part (iii). We are going to prove it only for the difference quotient $d_{h, k}^{+}$. The proof for $d_{h, k}^{-}$is fully analogous.

We are still assuming that $Q=\left(-\frac{1}{2}, \frac{1}{2}\right)^{n}$ and $\varrho$ is as above. Moreover, suppose that $f \in C_{0,0}^{\infty}(Q), \mathbf{x} \in Q$ and $h>0$. We get the following representation:

$$
\begin{aligned}
d_{h, k}^{+}(\mathcal{B} f)(\mathbf{x})= & \frac{1}{h} \int_{Q} f(\mathbf{y}) \frac{\mathbf{x}+h \mathbf{e}^{k}-\mathbf{y}}{\left|\mathbf{x}+h \mathbf{e}^{k}-\mathbf{y}\right|^{n}} \int_{\left|\mathbf{x}+h \mathbf{e}^{k}-\mathbf{y}\right|}^{\infty} \varrho\left(\mathbf{y}+\xi \frac{\mathbf{x}+h \mathbf{e}^{k}-\mathbf{y}}{\left|\mathbf{x}+h \mathbf{e}^{k}-\mathbf{y}\right|}\right) \xi^{n-1} \mathrm{~d} \xi \mathrm{d} \mathbf{y} \\
& -\frac{1}{h} \int_{Q} f(\mathbf{y}) \frac{\mathbf{x}-\mathbf{y}}{|\mathbf{x}-\mathbf{y}|^{n}} \int_{|\mathbf{x}-\mathbf{y}|}^{\infty} \varrho\left(\mathbf{y}+\xi \frac{\mathbf{x}-\mathbf{y}}{|\mathbf{x}-\mathbf{y}|}\right) \xi^{n-1} \mathrm{~d} \xi \mathrm{d} \mathbf{y} \\
= & \frac{1}{h} \int_{Q-h \mathbf{e}^{k}} f\left(\mathbf{y}+h \mathbf{e}^{k}\right) \frac{\mathbf{x}-\mathbf{y}}{|\mathbf{x}-\mathbf{y}|^{n}} \int_{|\mathbf{x}-\mathbf{y}|}^{\infty} \varrho\left(\mathbf{y}+h \mathbf{e}^{k}+\xi \frac{\mathbf{x}-\mathbf{y}}{|\mathbf{x}-\mathbf{y}|}\right) \xi^{n-1} \mathrm{~d} \xi \mathrm{d} \mathbf{y} \\
& -\frac{1}{h} \int_{Q} f(\mathbf{y}) \frac{\mathbf{x}-\mathbf{y}}{|\mathbf{x}-\mathbf{y}|^{n}} \int_{|\mathbf{x}-\mathbf{y}|}^{\infty} \varrho\left(\mathbf{y}+\xi \frac{\mathbf{x}-\mathbf{y}}{|\mathbf{x}-\mathbf{y}|}\right) \xi^{n-1} \mathrm{~d} \xi \mathrm{d} \mathbf{y} \\
= & \int_{Q-h \mathbf{e}^{k}} d_{h, k}^{+} f(\mathbf{y}) \frac{\mathbf{x}-\mathbf{y}}{|\mathbf{x}-\mathbf{y}|^{n}} \int_{|\mathbf{x}-\mathbf{y}|}^{\infty} \varrho\left(\mathbf{y}+h \mathbf{e}^{k}+\xi \frac{\mathbf{x}-\mathbf{y}}{|\mathbf{x}-\mathbf{y}|}\right) \xi \xi^{n-1} \mathrm{~d} \xi \mathrm{d} \mathbf{y} \\
& +\frac{1}{h} \int_{\left(Q-h \mathbf{e}^{k}\right) \backslash Q} f(\mathbf{y}) \frac{\mathbf{x}-\mathbf{y}}{|\mathbf{x}-\mathbf{y}|^{n}} \int_{|\mathbf{x}-\mathbf{y}|}^{\infty} \varrho\left(\mathbf{y}+h \mathbf{e}^{k}+\xi \frac{\mathbf{x}-\mathbf{y}}{|\mathbf{x}-\mathbf{y}|}\right) \xi^{n-1} \mathrm{~d} \xi \mathrm{d} \mathbf{y} \\
& -\frac{1}{h} \int_{Q \backslash\left(Q-h \mathbf{e}^{k}\right)} f(\mathbf{y}) \frac{\mathbf{x}-\mathbf{y}}{|\mathbf{x}-\mathbf{y}|^{n}} \int_{|\mathbf{x}-\mathbf{y}|}^{\infty} \varrho\left(\mathbf{y}+h \mathbf{e}^{k}+\xi \frac{\mathbf{x}-\mathbf{y}}{|\mathbf{x}-\mathbf{y}|}\right) \xi^{n-1} \mathrm{~d} \xi \mathrm{d} \mathbf{y} \\
& +\int_{Q} f(\mathbf{y}) \frac{\mathbf{x}-\mathbf{y}}{|\mathbf{x}-\mathbf{y}|^{n}} \int_{|\mathbf{x}-\mathbf{y}|}^{\infty} d_{h, k}^{+} \varrho\left(\mathbf{y}+\xi \frac{\mathbf{x}-\mathbf{y}}{|\mathbf{x}-\mathbf{y}|}\right) \xi^{n-1} \mathrm{~d} \xi \mathrm{d} \mathbf{y} \\
= & \int_{Q \cup\left(Q-h \mathbf{e}^{k}\right)} d_{h, k}^{+} f(\mathbf{y}) \frac{\mathbf{x}-\mathbf{y}}{|\mathbf{x}-\mathbf{y}|^{n}} \int_{|\mathbf{x}-\mathbf{y}|}^{\infty} \varrho\left(\mathbf{y}+h \mathbf{e}^{k}+\xi \frac{\mathbf{x}-\mathbf{y}}{|\mathbf{x}-\mathbf{y}|}\right) \xi^{n-1} \mathrm{~d} \xi \mathrm{d} \mathbf{y}
\end{aligned}
$$




$$
\begin{aligned}
& +\int_{Q} f(\mathbf{y}) \frac{\mathbf{x}-\mathbf{y}}{|\mathbf{x}-\mathbf{y}|^{n}} \int_{|\mathbf{x}-\mathbf{y}|}^{\infty} d_{h, k}^{+} \varrho\left(\mathbf{y}+\xi \frac{\mathbf{x}-\mathbf{y}}{|\mathbf{x}-\mathbf{y}|}\right) \xi^{n-1} \mathrm{~d} \xi \mathrm{d} \mathbf{y} \\
= & : \mathbf{a}^{h, 1}(\mathbf{x})+\mathbf{a}^{h, 2}(\mathbf{x}) .
\end{aligned}
$$

To get the fourth equality we used the fact that supp $f \subset Q$. A simple observation yields

$$
\left\|\varrho\left(\cdot+h \mathbf{e}^{k}\right)\right\|_{1, \infty}=\|\varrho\|_{1, \infty} \text { for all } h>0 .
$$

Let $i, j \in\{1, \ldots, n\}$ and assume that $h \in\left(0, \frac{1}{4}\right)$. Then the set $Q \cup\left(Q-h \mathbf{e}^{k}\right)$ is convex and it contains supp $\varrho\left(\cdot+h \mathbf{e}^{k}\right)$. Hence, we may use [16, p. 119] to get the following representation:

$$
\begin{aligned}
\partial_{j} a_{i}^{h, 1}(\mathbf{x})= & \text { p.v. } \int_{Q \cup\left(Q-h \mathbf{e}^{k}\right)} d_{h, k}^{+} f(\mathbf{y}) K_{i j}^{h}(\mathbf{x}, \mathbf{y}) \mathrm{d} \mathbf{y} \\
& +\int_{Q \cup\left(Q-h \mathbf{e}^{k}\right)} d_{h, k}^{+} f(\mathbf{y}) G_{i j}^{h}(\mathbf{x}, \mathbf{y}) \mathrm{d} \mathbf{y} \\
& +d_{h, k}^{+} f(\mathbf{x}) \int_{Q \cup\left(Q-h \mathbf{e}^{k}\right)} \frac{\left(x_{i}-y_{i}\right)\left(x_{j}-y_{j}\right)}{|\mathbf{x}-\mathbf{y}|^{2}} \varrho\left(\mathbf{y}+h \mathbf{e}^{k}\right) \mathrm{d} \mathbf{y} .
\end{aligned}
$$

In here, $G_{i j}$ is a kernel such that $\left|G_{i j}^{h}(\mathbf{x}, \mathbf{y})\right| \leq c\|\varrho\|_{1, \infty}|\mathbf{x}-\mathbf{y}|^{1-n}$ for all $\mathbf{x}, \mathbf{y} \in Q \cup\left(Q-h \mathbf{e}^{k}\right)$, $\mathbf{x} \neq \mathbf{y}$. The positive constant $c$ here depends only on $n$. We have also used (4.9) here. Furthermore, the kernel $K_{i, j}^{h}$ is expressed as

$$
\begin{aligned}
K_{i j}^{h}(\mathbf{x}, \mathbf{y}):= & \frac{\delta_{i j}}{|\mathbf{x}-\mathbf{y}|^{n}} \int_{0}^{\infty} \varrho\left(\mathbf{x}+h \mathbf{e}^{k}+\xi \frac{\mathbf{x}-\mathbf{y}}{|\mathbf{x}-\mathbf{y}|}\right) \xi^{n-1} \mathrm{~d} \xi \\
& +\frac{x_{i}-y_{i}}{|\mathbf{x}-\mathbf{y}|^{n+1}} \int_{0}^{\infty} \partial_{j} \varrho\left(\mathbf{x}+h \mathbf{e}^{k}+\xi \frac{\mathbf{x}-\mathbf{y}}{|\mathbf{x}-\mathbf{y}|}\right) \xi^{n} \mathrm{~d} \xi
\end{aligned}
$$

for any $\mathbf{x}, \mathbf{y} \in \mathbb{R}^{n}, \mathbf{x} \neq \mathbf{y}$. From the restriction $h \in\left(0, \frac{1}{4}\right)$ it follows that

$$
Q \cup\left(Q-h \mathbf{e}_{k}\right) \subset E:=Q \cup\left(Q-\frac{1}{4} \mathbf{e}_{k}\right) .
$$

In $[10$, Lemma 6.1$]$ it is proved that there exist constants $\kappa_{1}, \kappa_{2} \in(0, \infty)$ such that for every $h>0$ it holds that $K_{i j}^{h} \in \mathrm{SK}\left(E, \kappa_{1}\right) \cap \mathrm{CZ}\left(E, \kappa_{2}\right)$. The constants $\kappa_{1}, \kappa_{2}$ depend on $n$ and $\left\|\varrho\left(\cdot+h \mathbf{e}^{k}\right)\right\|_{1, \infty}$. From (4.9) it follows that $\kappa_{1}, \kappa_{2}$ are again independent of $h$.

Theorem 3.4 now grants the existence of a positive nondecreasing function $\widetilde{c}_{p, 1}$ such that for all $f \in C_{0,0}^{\infty}(Q)$ and all $h \in\left(0, \frac{1}{4}\right)$ the inequality

$$
\begin{aligned}
& \left(\int_{Q} \mid \text { p.v. }\left.\int_{Q \cup\left(Q-h \mathbf{e}^{k}\right)} d_{h, k}^{+} f(\mathbf{y}) K_{i j}^{h}(\mathbf{x}, \mathbf{y}) \mathrm{d} \mathbf{y}\right|^{p} \omega(\mathbf{x}) \mathrm{d} \mathbf{x}\right)^{\frac{1}{p}} \\
& \leq \widetilde{c}_{p, 1}\left([\omega]_{A_{p}}\right)\left(\int_{Q \cup\left(Q-h \mathbf{e}^{k}\right)}\left|d_{h, k}^{+} f(\mathbf{x})\right|^{p} \omega(\mathbf{x}) \mathrm{d} \mathbf{x}\right)^{\frac{1}{p}} .
\end{aligned}
$$

holds true. In the same way as in part (ii), there exists a positive constant $C$ such that

$$
\begin{aligned}
& \left(\int_{Q}\left|\int_{Q \cup\left(Q-h \mathbf{e}^{k}\right)} d_{h, k}^{+} f(\mathbf{y}) G_{i j}^{h}(\mathbf{x}, \mathbf{y}) \mathrm{d} \mathbf{y}\right|^{p} \omega(\mathbf{x}) \mathrm{d} \mathbf{x}\right)^{\frac{1}{p}} \\
& \quad \leq C[\omega]_{A_{p}}^{\frac{1}{p-1}}\|\varrho\|_{1, \infty}\left(\int_{Q \cup\left(Q-h \mathbf{e}^{k}\right)}\left|d_{h, k}^{+} f(\mathbf{x})\right|^{p} \omega(\mathbf{x}) \mathrm{d} \mathbf{x}\right)^{\frac{1}{p}}
\end{aligned}
$$


and

$$
\begin{aligned}
& \left(\int_{Q}\left|d_{h, k}^{+} f(\mathbf{y}) \int_{Q \cup\left(Q-h \mathbf{e}^{k}\right)} \frac{\left(x_{i}-y_{i}\right)\left(x_{j}-y_{j}\right)}{|\mathbf{x}-\mathbf{y}|^{2}} \varrho\left(\mathbf{y}+h \mathbf{e}^{k}\right) \mathrm{d} \mathbf{y}\right|^{p} \omega(\mathbf{x}) \mathrm{d} \mathbf{x}\right)^{\frac{1}{p}} \\
& \quad \leq\|\varrho\|_{1}\left(\int_{Q}\left|d_{h, k}^{+} f(\mathbf{x})\right|^{p} \omega(\mathbf{x}) \mathrm{d} \mathbf{x}\right)^{\frac{1}{p}}
\end{aligned}
$$

both hold for all $f \in C_{0,0}^{\infty}(Q)$ and all $h \in\left(0, \frac{1}{4}\right)$. It follows from the obtained estimates that there exists a positive nondecreasing function $\widetilde{C}_{p, 1}$ such that

$$
\left(\int_{Q}\left|\partial_{j} a_{i}^{h, 1}(\mathbf{x})\right|^{p} \omega(\mathbf{x}) \mathrm{d} \mathbf{x}\right)^{\frac{1}{p}} \leq \widetilde{C}_{p, 1}\left([\omega]_{A_{p}}\right)\left(\int_{Q \cup\left(Q-h \mathbf{e}^{k}\right)}\left|d_{h, k}^{+} f(\mathbf{x})\right|^{p} \omega(\mathbf{x}) \mathrm{d} \mathbf{x}\right)^{\frac{1}{p}}
$$

holds for all $f \in C_{0,0}^{\infty}(Q)$ and all $h \in\left(0, \frac{1}{4}\right)$.

Let us proceed by estimating the partial derivatives of $\mathbf{a}^{2}(\mathbf{x})$, still under the assumption $h \in\left(0, \frac{1}{4}\right)$. Since supp $\varrho \subset\left(-\frac{1}{4}, \frac{1}{4}\right)^{n}$ holds, the function $d_{h, k}^{+} \varrho$ is supported in $Q$. The cube $Q$ is convex, thus we may again use the same calculation as in [16, p. 119] to obtain

$$
\begin{aligned}
\partial_{j} a_{i}^{h, 2}(\mathbf{x})=\text { p.v. } & \int_{Q} f(\mathbf{y}) M_{i j}^{h}(\mathbf{x}, \mathbf{y}) \mathrm{d} \mathbf{y}+\int_{Q} f(\mathbf{y}) H_{i j}^{h}(\mathbf{x}, \mathbf{y}) \mathrm{d} \mathbf{y} \\
& +f(\mathbf{x}) \int_{Q} \frac{\left(x_{i}-y_{i}\right)\left(x_{j}-y_{j}\right)}{|\mathbf{x}-\mathbf{y}|^{2}} d_{h, k}^{+} \varrho(\mathbf{y}) \mathrm{d} \mathbf{y} .
\end{aligned}
$$

In here, $H_{i j}^{h}$ is a kernel satisfying $\left|H_{i j}^{h}(\mathbf{x}, \mathbf{y})\right| \leq C|\mathbf{x}-\mathbf{y}|^{1-n}$ for all $\mathbf{x}, \mathbf{y} \in Q, \mathbf{x} \neq \mathbf{y}$, with $C$ depending only on $n$ and $\|\varrho\|_{2, \infty}$. Furthermore, the kernel $M_{i j}^{h}$ is, for $\mathbf{x}, \mathbf{y} \in \mathbb{R}^{n}, \mathbf{x} \neq \mathbf{y}$, defined as follows:

$$
\begin{aligned}
M_{i j}^{h}(\mathbf{x}, \mathbf{y}):= & \frac{\delta_{i j}}{|\mathbf{x}-\mathbf{y}|^{n}} \int_{0}^{\infty} d_{h, k}^{+} \varrho\left(\mathbf{x}+\xi \frac{\mathbf{x}-\mathbf{y}}{|\mathbf{x}-\mathbf{y}|}\right) \xi^{n-1} \mathrm{~d} \xi \\
& +\frac{x_{i}-y_{i}}{|\mathbf{x}-\mathbf{y}|^{n+1}} \int_{0}^{\infty} d_{h, k}^{+} \partial_{j} \varrho\left(\mathbf{x}+\xi \frac{\mathbf{x}-\mathbf{y}}{|\mathbf{x}-\mathbf{y}|}\right) \xi^{n} \mathrm{~d} \xi \\
= & : m_{i j}^{h, 1}(\mathbf{x}, \mathbf{y})+m_{i j}^{h, 2}(\mathbf{x}, \mathbf{y}) .
\end{aligned}
$$

Observe that if $\mathbf{x}, \mathbf{y} \in Q$ and $\xi>n$, then

$$
\xi-h>n-\frac{1}{4}>\frac{\sqrt{n}}{4}=\operatorname{diam}(\operatorname{supp} \varrho),
$$

and therefore

$$
\mathbf{x}+\xi \frac{\mathbf{x}-\mathbf{y}}{|\mathbf{x}-\mathbf{y}|} \notin \operatorname{supp} \varrho \text { and } \mathbf{x}+h \mathbf{e}^{k}+\xi \frac{\mathbf{x}-\mathbf{y}}{|\mathbf{x}-\mathbf{y}|} \notin \operatorname{supp} \varrho .
$$

Hence, the integrals over $(0, \infty)$ in the definition of $M_{i j}^{h}$ may be replaced by integrals over $(0, n)$.

From [10, Lemma 6.1] it follows that $m_{i j}^{h, 1}$ is a Calderón-Zygmund kernel with respect to $Q$, with a constant independent of $h$. Here, notice that the function $d_{h, k}^{+} \varrho$ plays the role of $\varrho$ in [10], and $\left\|d_{h, k}^{+} \varrho\right\|_{1, \infty} \leq\|\varrho\|_{2, \infty}$. In the next step, we shall verify that $m_{i j}^{h, 1}$ is a standard 
kernel with respect to $Q$ with a constant independent of $h$. Let $\mathbf{x}, \mathbf{y}, \mathbf{z} \in Q$ be such that $\mathbf{x} \neq \mathbf{y}$ and $|\mathbf{x}-\mathbf{z}| \leq \frac{1}{2}|\mathbf{x}-\mathbf{y}|$. It is easy to see that

$$
\left|m_{i j}^{1}(\mathbf{x}, \mathbf{y})\right| \leq \frac{\|\nabla \varrho\|_{\infty}}{|\mathbf{x}-\mathbf{y}|^{n}} \int_{0}^{n} \xi^{n-1} \mathrm{~d} \xi=\frac{\|\nabla \varrho\|_{\infty} n^{n-1}}{|\mathbf{x}-\mathbf{y}|^{n}} .
$$

Using (3.6), we obtain

$$
\left|\frac{1}{|\mathbf{x}-\mathbf{y}|^{n}}-\frac{1}{|\mathbf{y}-\mathbf{z}|^{n}}\right|=\frac{|| \mathbf{y}-\mathbf{z}|-| \mathbf{x}-\mathbf{y}||}{|\mathbf{x}-\mathbf{y}|^{n}|\mathbf{y}-\mathbf{z}|^{n}} \cdot \sum_{b=0}^{n-1}|\mathbf{y}-\mathbf{z}|^{b}|\mathbf{x}-\mathbf{y}|^{n-b-1} \leq \frac{2^{n} n|\mathbf{x}-\mathbf{z}|}{|\mathbf{x}-\mathbf{y}|^{n+1}}
$$

and

$$
\begin{aligned}
\left|\frac{x_{i}-y_{i}}{|\mathbf{x}-\mathbf{y}|^{n+1}}-\frac{z_{i}-y_{i}}{|\mathbf{z}-\mathbf{y}|^{n+1}}\right| & \leq \frac{\left|x_{i}-z_{i}\right|}{|\mathbf{x}-\mathbf{y}|^{n+1}}+\left|y_{i}-z_{i}\right|\left|\frac{1}{|\mathbf{x}-\mathbf{y}|^{n+1}}-\frac{1}{|\mathbf{z}-\mathbf{y}|^{n+1}}\right| \\
& \leq\left(1+3 \cdot 2^{n}(n+1)\right) \frac{|\mathbf{x}-\mathbf{z}|}{|\mathbf{x}-\mathbf{y}|^{n+1}}
\end{aligned}
$$

Moreover, we have

$$
\left|\frac{\mathbf{x}-\mathbf{y}}{|\mathbf{x}-\mathbf{y}|}-\frac{\mathbf{z}-\mathbf{y}}{|\mathbf{z}-\mathbf{y}|}\right|=\left|\frac{\mathbf{x}-\mathbf{z}}{|\mathbf{x}-\mathbf{y}|}+(\mathbf{z}-\mathbf{y}) \frac{|\mathbf{z}-\mathbf{y}|-|\mathbf{x}-\mathbf{y}|}{|\mathbf{x}-\mathbf{y}||\mathbf{z}-\mathbf{y}|}\right| \leq 2 \frac{|\mathbf{x}-\mathbf{z}|}{|\mathbf{x}-\mathbf{y}|} .
$$

Now we use (4.12), (4.14), Lemma 4.3 and the inequality

$$
|\mathbf{x}-\mathbf{z}| \leq \frac{\operatorname{diam} Q|\mathbf{x}-\mathbf{z}|}{|\mathbf{x}-\mathbf{y}|}=\frac{2 \sqrt{n}|\mathbf{x}-\mathbf{z}|}{|\mathbf{x}-\mathbf{y}|}
$$

to get

$$
\begin{aligned}
& \left|m_{i j}^{h, 1}(\mathbf{x}, \mathbf{y})-m_{i j}^{h, 1}(\mathbf{z}, \mathbf{y})\right| \\
& \leq\left|\frac{1}{|\mathbf{x}-\mathbf{y}|^{n}}-\frac{1}{|\mathbf{y}-\mathbf{z}|^{n}}\right| \int_{0}^{n}\left|d_{h, k}^{+} \varrho\left(\mathbf{z}+\xi \frac{\mathbf{z}-\mathbf{y}}{|\mathbf{z}-\mathbf{y}|}\right)\right| \xi^{n-1} \mathrm{~d} \xi \\
& \quad+\frac{1}{|\mathbf{x}-\mathbf{y}|^{n}} \int_{0}^{n}\left|d_{h, k}^{+} \varrho\left(\mathbf{x}+\xi \frac{\mathbf{x}-\mathbf{y}}{|\mathbf{x}-\mathbf{y}|}\right)-d_{h, k}^{+} \varrho\left(\mathbf{z}+\xi \frac{\mathbf{z}-\mathbf{y}}{|\mathbf{z}-\mathbf{y}|}\right)\right| \xi^{n-1} \mathrm{~d} \xi \\
& \leq(2 n)^{n}\|\nabla \varrho\|_{\infty} \frac{|\mathbf{x}-\mathbf{z}|}{|\mathbf{x}-\mathbf{y}|^{n+1}}+\frac{\left\|\nabla^{2} \varrho\right\|_{\infty}}{|\mathbf{x}-\mathbf{y}|^{n}} \int_{0}^{n}\left(|\mathbf{x}-\mathbf{z}|+2 \xi \frac{|\mathbf{x}-\mathbf{z}|}{|\mathbf{x}-\mathbf{y}|}\right) \xi^{n-1} \mathrm{~d} \xi \\
& \quad \leq\left(2^{n}+4\right) n^{n}\|\varrho\|_{2, \infty} \frac{|\mathbf{x}-\mathbf{z}|}{|\mathbf{x}-\mathbf{y}|^{n+1}} .
\end{aligned}
$$

Analogously, we obtain the following:

$$
\begin{aligned}
& \left|m_{i j}^{h, 1}(\mathbf{y}, \mathbf{x})-m_{i j}^{h .1}(\mathbf{y}, \mathbf{z})\right| \\
& \leq\left|\frac{1}{|\mathbf{x}-\mathbf{y}|^{n}}-\frac{1}{|\mathbf{y}-\mathbf{z}|^{n}}\right| \int_{0}^{n}\left|d_{h, k}^{+} \varrho\left(\mathbf{y}+\xi \frac{\mathbf{y}-\mathbf{z}}{|\mathbf{y}-\mathbf{z}|}\right)\right| \xi^{n-1} \mathrm{~d} \xi \\
& \quad+\frac{1}{|\mathbf{x}-\mathbf{y}|^{n}} \int_{0}^{n}\left|d_{h, k}^{+} \varrho\left(\mathbf{y}+\xi \frac{\mathbf{y}-\mathbf{x}}{|\mathbf{y}-\mathbf{x}|}\right)-d_{h, k}^{+} \varrho\left(\mathbf{y}+\xi \frac{\mathbf{y}-\mathbf{z}}{|\mathbf{y}-\mathbf{z}|}\right)\right| \xi^{n-1} \mathrm{~d} \xi \\
& \quad \leq 2^{n+1} n^{n}\|\varrho\|_{2, \infty} \frac{|\mathbf{x}-\mathbf{z}|}{|\mathbf{x}-\mathbf{y}|^{n+1}} .
\end{aligned}
$$


Therefore, $m_{i j}^{h, 1}$ is a standard kernel with respect to $Q$ with a constant independent of $h$. Analogously, using (3.6), (4.13) and (4.14), we show that $m_{i j}^{h, 2}$ is a standard kernel with respect to $Q$ with a constant depending only on $n$ and $\|\varrho\|_{3, \infty}$. Hence, $M_{i j}^{h}$ is a standard kernel with respect to $Q$ with a constant independent of $h$. By Theorem 3.4 there exists a positive nondecreasing function $\widetilde{c}_{p, 2}$ such that for all $h \in\left(0, \frac{1}{4}\right)$ and all $f \in C_{0,0}^{\infty}(Q)$ we have

$$
\left(\int_{Q} \mid \text { p.v. }\left.\int_{Q} f(\mathbf{y}) M_{i j}^{h}(\mathbf{x}, \mathbf{y}) \mathrm{d} \mathbf{y}\right|^{p} \omega(\mathbf{x}) \mathrm{d} \mathbf{x}\right)^{\frac{1}{p}} \leq \widetilde{c}_{p, 2}\left([\omega]_{A_{p}}\right)\left(\int_{Q}|f(\mathbf{x})|^{p} \omega(\mathbf{x}) \mathrm{d} \mathbf{x}\right)^{\frac{1}{p}} .
$$

The remaining parts of (4.11) are treated analogously as their counterparts in $\frac{\partial a_{j}^{1}}{\partial x_{i}}$. Altogether, it follows that there exists a positive nondecreasing function $\widetilde{C}_{p, 2}$ such that

$$
\left(\int_{Q}\left|\frac{\partial}{\partial x_{j}} a_{i}^{h, 2}(\mathbf{x})\right|^{p} \omega(\mathbf{x}) \mathrm{d} \mathbf{x}\right)^{\frac{1}{p}} \leq \widetilde{C}_{p, 2}\left([\omega]_{A_{p}}\right)\left(\int_{Q}|f(\mathbf{x})|^{p} \omega(\mathbf{x}) \mathrm{d} \mathbf{x}\right)^{\frac{1}{p}}
$$

holds for all $h \in\left(0, \frac{1}{4}\right)$ and $f \in C_{0,0}^{\infty}(Q)$. Using this result and (4.10), we obtain the existence of a positive nondecreasing function $\widetilde{C}_{p}$ such that the following holds for all $h \in\left(0, \frac{1}{4}\right)$ and all $f \in C_{0,0}^{\infty}(Q)$ :

$$
\begin{aligned}
\left(\int_{Q}\left|d_{h, k}^{+} \nabla(\mathcal{B} f)(\mathbf{x})\right|^{p} \omega(\mathbf{x}) \mathrm{d} \mathbf{x}\right)^{\frac{1}{p}} & \leq \widetilde{C}_{p}\left([\omega]_{A_{p}}\right)\left(\int_{Q}\left(\left|\nabla \mathbf{a}^{h, 1}(\mathbf{x})\right|+\left|\nabla \mathbf{a}^{h, 2}(\mathbf{x})\right|\right)^{p} \omega(\mathbf{x}) \mathrm{d} \mathbf{x}\right)^{\frac{1}{p}} \\
& \leq \widetilde{C}_{p}\left([\omega]_{A_{p}}\right)\left(\int_{Q \cup\left(Q-h \mathbf{e}^{k}\right)}\left(\left|d_{h, k}^{+} f(\mathbf{x})\right|+|f(\mathbf{x})|\right)^{p} \omega(\mathbf{x}) \mathrm{d} \mathbf{x}\right)^{\frac{1}{p}} \\
& \leq \widetilde{C}_{p}\left([\omega]_{A_{p}}\right)\left(\int_{Q}\left(\left|d_{h, k}^{+} f(\mathbf{x})\right|+\left|d_{h, k}^{-} f(\mathbf{x})\right|+|f(\mathbf{x})|\right)^{p} \omega(\mathbf{x}) \mathrm{d} \mathbf{x}\right)^{\frac{1}{p}} .
\end{aligned}
$$

As next, assume that $h \in\left[\frac{1}{4}, \infty\right)$. Let $C_{p}$ be the function obtained in part (ii). By (4.2) we have

$$
\begin{aligned}
\left(\int_{Q}\left|d_{h, k}^{+} \nabla(\mathcal{B} f)(\mathbf{x})\right|^{p} \omega(\mathbf{x}) \mathrm{d} \mathbf{x}\right)^{\frac{1}{p}} & \leq C_{p}\left([\omega]_{A_{p}}\right)\left(\int_{Q}\left|\frac{\nabla(\mathcal{B} f)\left(\mathbf{x}+h \mathbf{e}^{k}\right)}{h}-\frac{\nabla(\mathcal{B} f)(\mathbf{x})}{h}\right|^{p} \omega(\mathbf{x}) \mathrm{d} \mathbf{x}\right)^{\frac{1}{p}} \\
& \leq 4 C_{p}\left([\omega]_{A_{p}}\right)\left(\int_{Q}\left(\left|\nabla(\mathcal{B} f)\left(\mathbf{x}+h \mathbf{e}^{k}\right)\right|+|\nabla(\mathcal{B} f)(\mathbf{x})|\right)^{p} \omega(\mathbf{x}) \mathrm{d} \mathbf{x}\right)^{\frac{1}{p}} \\
& \leq 8 C_{p}\left([\omega]_{A_{p}}\right)\left(\int_{Q}|\nabla(\mathcal{B} f)(\mathbf{x})|^{p} \omega(\mathbf{x}) \mathrm{d} \mathbf{x}\right)^{\frac{1}{p}} \\
& \leq 8 C_{p}\left([\omega]_{A_{p}}\right)\left(\int_{Q}|f(\mathbf{x})|^{p} \omega(\mathbf{x}) \mathrm{d} \mathbf{x}\right)^{\frac{1}{p}}
\end{aligned}
$$

for all $f \in C_{0,0}^{\infty}(Q)$ and $h \geq \frac{1}{4}$. In the third step we have used the fact that $\nabla(\mathcal{B} f)(\mathbf{x})=0$ for $\mathbf{x} \in \mathbb{R}^{n} \backslash Q$.

At this point we have proved that if $Q=\left(-\frac{1}{2}, \frac{1}{2}\right)^{n}$, then there exist positive nondecreasing functions $C_{p}, \widetilde{C}_{p}$ such that for all $f \in C_{0,0}^{\infty}(Q)$ and all $h>0$ the inequalities (4.2) and

$$
\left(\int_{Q}\left|d_{h, k}^{+} \nabla(\mathcal{B} f)(\mathbf{x})\right|^{p} \omega(\mathbf{x}) \mathrm{d} \mathbf{x}\right)^{\frac{1}{p}}
$$




$$
\leq \widetilde{C}_{p}\left([\omega]_{A_{p}}\right)\left(\int_{Q}\left(\left|d_{h, k}^{+} f(\mathbf{x})\right|+\left|d_{h, k}^{-} f(\mathbf{x})\right|+|f(\mathbf{x})|\right)^{p} \omega(\mathbf{x}) \mathrm{d} \mathbf{x}\right)^{\frac{1}{p}}
$$

are satisfied. In here, the operator $\mathcal{B}$ is defined by (4.5). Now consider the cube $\lambda Q=$ $\left(-\frac{\lambda}{2}, \frac{\lambda}{2}\right)^{n}$ with a fixed $\lambda>0$. Define $\varrho_{\lambda}(\mathbf{x}):=\lambda^{-n} \varrho(\mathbf{x})$ for $\mathbf{x} \in \lambda Q$. Then $\varrho_{\lambda} \in C_{0}^{\infty}(\lambda Q)$ and $\int_{\lambda Q} \varrho_{\lambda}(\mathbf{x}) \mathrm{d} \mathbf{x}=1$. For any $f \in C_{0,0}^{\infty}(\lambda Q)$ define $\mathcal{B}_{\lambda} f$ by the formula (4.5), where $Q$ and $\varrho$ are replaced by $\lambda Q$ and $\varrho_{\lambda}$, respectively. Similarly as before one verifies that $\operatorname{div} \mathcal{B}_{\lambda} f=f$ holds a.e. in $\lambda Q$. Using the proven result for $Q$ and rescaling through the change of variables $\mathbf{x} \rightarrow \lambda \mathbf{x}$, we obtain

$$
\left(\int_{\lambda Q}\left|\nabla\left(\mathcal{B}_{\lambda} f\right)(\mathbf{x})\right|^{p} \omega(\mathbf{x}) \mathrm{d} \mathbf{x}\right)^{\frac{1}{p}} \leq C_{p}\left([\omega]_{A_{p}}\right)\left(\int_{\lambda Q}|f(\mathbf{x})|^{p} \omega(\mathbf{x}) \mathrm{d} \mathbf{x}\right)^{\frac{1}{p}}
$$

and

$$
\begin{aligned}
& \left(\int_{\lambda Q}\left|d_{h, k}^{ \pm} \nabla\left(\mathcal{B}_{\lambda} f\right)(\mathbf{x})\right|^{p} \omega(\mathbf{x}) \mathrm{d} \mathbf{x}\right)^{\frac{1}{p}} \\
& \leq \widetilde{C}_{p}\left([\omega]_{A_{p}}\right)\left(\int_{\lambda Q}\left(\left|d_{h, k}^{+} f(\mathbf{x})\right|+\left|d_{h, k}^{-} f(\mathbf{x})\right|+\frac{|f(\mathbf{x})|}{\lambda}\right)^{p} \omega(\mathbf{x}) \mathrm{d} \mathbf{x}\right)^{\frac{1}{p}} .
\end{aligned}
$$

Notice also that $\lambda=\ell(\lambda Q)$. Obviously, this result remains unchanged when the cube $\lambda Q$ is shifted, e.g., replaced by $\mathbf{x}_{0}+\lambda Q$ for any fixed $\mathbf{x}_{0} \in \mathbb{R}^{n}$. Hence, we have now proved (i)-(iii) in full generality.

Part (iv) is proven by a standard approximation argument, using density of $C_{0,0}^{\infty}(Q)$ in $L_{\omega, 0}^{p}(Q)$ for any fixed $p$ and $\omega$. This completes the proof.

Remark 4.4 Corollary 4.2 follows now from Theorems 3.1 and 4.1.

\section{Interior regularity}

From now on, let $\Omega \subset \mathbb{R}^{n}$ be a bounded domain. We also assume that $\mathbf{S}$ has a $(p, \delta)$-structure for some $p \in(1, \infty)$ and $\delta \geq 0$. The properties of $\mathbf{S}$ and the standard theory of monotone operators imply in a standard way the existence of a unique $\mathbf{u} \in W_{0 \text {,div }}^{1, p}(\Omega)$, satisfying

$$
\int_{\Omega} \mathbf{S}(\mathbf{D u}) \cdot \mathbf{D w} \mathrm{d} \mathbf{x}=\int_{\Omega} \mathbf{f} \cdot \mathbf{w} \mathrm{d} \mathbf{x}
$$

for all $\mathbf{w} \in W_{0, \text { div }}^{1, p}(\Omega)$, i.e., $\mathbf{u}$ is a weak solution of (1.1). By choosing $\mathbf{w}=\mathbf{u}$ in (5.1) and using Proposition 2.8, the properties of $\mathbf{S}$, the Poincaré inequality (cf. [25, Lemma 3]), Korn inequality (cf. [13, Theorem 6.10]) and Young inequality (2.1), we obtain that this solution satisfies the a priori estimate

$$
\gamma_{0}(p) \int_{\Omega} \varphi(|\nabla \mathbf{u}|) \mathrm{d} \mathbf{x} \leq c \int_{\Omega} \varphi^{*}(|\mathbf{f}|) \mathrm{d} \mathbf{x} .
$$

From here on, we denote by $\gamma_{i}(p), i=0,1$, the constants in Definition 2.6 for a given $p$. Moreover, all constants may depend on the characteristics of $\mathbf{S}, \operatorname{diam}(\Omega),|\Omega|$, the space dimension $n$ and on the John constants of $\Omega$. The dependence on these quantities will not be mentioned explicitly anymore. However, dependence on other quantities will be specified. 
Theorem 5.1 Let the extra stress tensor $\mathbf{S}$ have a $(p, \delta)$-structure for some $p \in(1,2]$ and $\delta \in[0, \infty)$, and let $\mathbf{F}$ be the associated tensor field to $\mathbf{S}$. Let $\Omega \subset \mathbb{R}^{n}$ be a bounded domain and let $\mathbf{f} \in L^{\varphi^{*}}(\Omega)$. Let $Q \subseteq \Omega$ be a cube and let $\xi \in C_{0}^{\infty}(\Omega)$ satisfy $\chi_{\frac{1}{2} Q} \leq \xi \leq \chi_{\frac{3}{4}} Q$. Then the unique weak solution $\mathbf{u} \in W_{0 \text {, div }}^{1, p}(\Omega)$ of the problem (5.1) satisfies

$$
\int_{\frac{1}{2} Q}|\nabla \mathbf{F}(\mathbf{D u})|^{2} \mathrm{~d} \mathbf{x} \leq c\left(\|\xi\|_{2, \infty}\right) \int_{Q} \varphi^{*}(|\mathbf{f}|)+\varphi(|\nabla \mathbf{u}|) \mathrm{d} \mathbf{x} .
$$

Proof Let $k \in\{1, \ldots, n\}$. We would like to use $\mathbf{w}=d_{h, k}^{-}\left(\xi^{2} d_{h, k}^{+} \mathbf{u}\right)$ as a test function in (5.1). However, this is not possible since $\operatorname{div} \mathbf{w} \neq 0$. Instead, we start by using Corollary 4.2 , where $Q$ is replaced by $\frac{3}{4} Q$, with the setting

$$
f:=\operatorname{div}\left(\xi^{2} d_{h, k}^{+} \mathbf{u}\right), \quad h<\frac{1}{4} \ell(Q), \quad \psi=\varphi .
$$

It provides a function $\mathbf{v} \in W_{0}^{1, \varphi}\left(\frac{3}{4} Q\right)$ which solves

$$
\begin{aligned}
\operatorname{div} \mathbf{v} & =\operatorname{div}\left(\xi^{2} d_{h, k}^{+} \mathbf{u}\right) & & \text { in } \frac{3}{4} Q, \\
\mathbf{v} & =\mathbf{0} & & \text { on } \partial \frac{3}{4} Q,
\end{aligned}
$$

and satisfies the corresponding estimates in Corollary 4.2. Therefore, ${ }^{2}$ the vector field $\mathbf{w}=$ $d_{h, k}^{-}\left(\xi^{2} d_{h, k}^{+} \mathbf{u}-\mathbf{v}\right)$ belongs to $W_{0, \operatorname{div}}^{1, \varphi}(\Omega)$ and from (5.1) we get

$$
\begin{aligned}
& \int_{\Omega} \xi^{2} d_{h, k}^{+} \mathbf{S}(\mathbf{D u}) \cdot d_{h, k}^{+} \mathbf{D u} \mathrm{d} \mathbf{x} \\
& =\int_{\Omega} \mathbf{S}(\mathbf{D u}) \cdot \mathbf{D} d_{h, k}^{-} \mathbf{v}-\mathbf{S}(\mathbf{D u}) \cdot d_{h, k}^{-}\left(2 \xi \nabla \xi \stackrel{s}{\otimes} d_{h, k}^{+} \mathbf{u}\right) \mathrm{d} \mathbf{x} \\
& \quad+\int_{\Omega} \mathbf{f} \cdot d_{h, k}^{-}\left(\xi^{2} d_{h, k}^{+} \mathbf{u}\right)-\mathbf{f} \cdot d_{h, k}^{-} \mathbf{v} \mathrm{d} \mathbf{x}=: \sum_{j=1}^{4} I_{j} .
\end{aligned}
$$

The term providing the information concerning the regularity of the solution is the integral on the left-hand side. Indeed, from (2.18) one gets

$$
\int_{\Omega} \xi^{2}\left|d_{h, k}^{+} \mathbf{F}(\mathbf{D u})\right|^{2} \mathrm{~d} \mathbf{x} \leq c \int_{\Omega} \xi^{2} d_{h, k}^{+} \mathbf{S}(\mathbf{D u}) \cdot d_{h, k}^{+} \mathbf{D u} \mathrm{d} \mathbf{x} .
$$

In the same way as in [4, Lemma 3.11] one can show that

$$
\int_{\Omega} \varphi\left(\xi\left|\nabla d_{h, k}^{ \pm} \mathbf{u}\right|\right) \mathrm{d} \mathbf{x} \leq c \int_{\Omega} \xi^{2}\left|d_{h, k}^{ \pm} \mathbf{F}(\mathbf{D u})\right|^{2} \mathrm{~d} \mathbf{x}+c\left(\|\xi\|_{1, \infty}\right) \int_{Q} \varphi(|\nabla \mathbf{u}|) \mathrm{d} \mathbf{x} .
$$

Combining the last two inequalities together with (5.3), we obtain the inequality

$$
\int_{\Omega} \xi^{2}\left|d_{h, k}^{+} \mathbf{F}(\mathbf{D u})\right|^{2}+\varphi\left(\xi\left|\nabla d_{h, k}^{+} \mathbf{u}\right|\right) \mathrm{d} \mathbf{x} \leq c \sum_{j=1}^{4}\left|I_{j}\right|+c\left(\|\xi\|_{1, \infty}\right) \int_{Q} \varphi(|\nabla \mathbf{u}|) \mathrm{d} \mathbf{x} .
$$

Now we are going to find an appropriate estimate for each of the terms $I_{j}$. At first, observe that if $\mathbf{Q} \in \mathbb{R}_{\mathrm{sym}}^{n \times n}$ and $t>0$, then (2.17) (with $\mathbf{P}=\mathbf{0}$ ) and (2.2) (recall that $\Delta_{2}(\varphi)<\infty$ ) yield

$$
|\mathbf{S}(\mathbf{Q})| t \leq c \varphi^{\prime}(|\mathbf{Q}|) t \leq c\left(\varepsilon^{-1}\right) \varphi(|\mathbf{Q}|)+\varepsilon \varphi(t) .
$$

${ }^{2}$ We extend $\mathbf{v}$ and $f$ by zero to the whole domain $\Omega$. 
Making use of (5.5), (2.4) and the $\Delta_{2}$-condition for $\varphi$, we have

$$
\begin{aligned}
\left|I_{2}\right| & \leq c\left(\varepsilon^{-1}\right) \int_{Q} \varphi(|\mathbf{D u}|) \mathrm{d} \mathbf{x}+\varepsilon \int_{\Omega} \varphi\left(\left|d_{h, k}^{-}\left(2 \xi \nabla \xi \stackrel{s}{\otimes} d_{h, k}^{+} \mathbf{u}\right)\right|\right) \mathrm{d} \mathbf{x} \\
& \leq c\left(\varepsilon^{-1}\right) \int_{Q} \varphi(|\mathbf{D u}|) \mathrm{d} \mathbf{x}+\varepsilon \int_{\Omega} \varphi\left(\left|\nabla\left(2 \xi \nabla \xi \stackrel{s}{\otimes} d_{h, k}^{+} \mathbf{u}\right)\right|\right) \mathrm{d} \mathbf{x} \\
& \leq c\left(\varepsilon^{-1},\|\xi\|_{2, \infty}\right) \int_{Q} \varphi(|\nabla \mathbf{u}|) \mathrm{d} \mathbf{x}+\varepsilon c\left(\|\xi\|_{1, \infty}\right) \int_{\Omega} \varphi\left(\left|\xi \nabla d_{h, k}^{+} \mathbf{u}\right|\right) \mathrm{d} \mathbf{x} .
\end{aligned}
$$

Next, inequality (2.1), the fact that $\Delta_{2}(\varphi)<\infty$ and $0 \leq \xi^{2} \leq \xi$, imply

$$
\begin{aligned}
\left|I_{3}\right| & \leq c\left(\varepsilon^{-1}\right) \int_{Q} \varphi^{*}(|\mathbf{f}|) \mathrm{d} \mathbf{x}+\varepsilon \int_{\Omega} \varphi\left(\left|d_{h, k}^{-}\left(\xi^{2} d_{h, k}^{+} \mathbf{u}\right)\right|\right) \mathrm{d} \mathbf{x} \\
& \leq c\left(\varepsilon^{-1}\right) \int_{Q} \varphi^{*}(|\mathbf{f}|) \mathrm{d} \mathbf{x}+\varepsilon \int_{\Omega} \varphi\left(\left|\nabla\left(\xi^{2} d_{h, k}^{+} \mathbf{u}\right)\right|\right) \mathrm{d} \mathbf{x} \\
& \leq c\left(\varepsilon^{-1},\|\xi\|_{1, \infty}\right) \int_{\Omega} \varphi^{*}(|\mathbf{f}|)+\varphi(|\nabla \mathbf{u}|) \mathrm{d} \mathbf{x}+\varepsilon c \int_{\Omega} \varphi\left(\left|\xi \nabla d_{h, k}^{+} \mathbf{u}\right|\right) \mathrm{d} \mathbf{x} .
\end{aligned}
$$

In order to use Corollary 4.2 we observe that

$$
\begin{aligned}
\left|d_{h, k}^{ \pm} \operatorname{div}\left(\xi^{2} d_{h, k}^{+} \mathbf{u}\right)\right| & \leq c\left(\|\xi\|_{2, \infty}\right) \xi\left|d_{h, k}^{+} \mathbf{u}\right|+c\left(\|\xi\|_{1, \infty}\right)\left|d_{h, k}^{ \pm}\left(\xi d_{h, k}^{+} \mathbf{u}\right)\right|, \\
\left|\operatorname{div}\left(\xi^{2} d_{h, k}^{+} \mathbf{u}\right)\right| & \leq c\left(\|\xi\|_{1, \infty}\right) \xi\left|d_{h, k}^{+} \mathbf{u}\right|,
\end{aligned}
$$

where we also used that $\mathbf{u}$ is solenoidal. Estimate (5.5), Corollary 4.2(iii), inequalities (5.6), (2.4), (2.3) and the condition $\Delta_{2}(\psi)<\infty$ now yield the following:

$$
\begin{aligned}
\left|I_{1}\right| \leq & c\left(\varepsilon^{-1}\right) \int_{Q} \varphi(|\mathbf{D u}|) \mathrm{d} \mathbf{x}+\varepsilon \int_{\Omega} \varphi\left(\left|\nabla d_{h, k}^{-} \mathbf{v}\right|\right) \mathrm{d} \mathbf{x} \\
\leq & c\left(\varepsilon^{-1}\right) \int_{Q} \varphi(|\mathbf{D u}|) \mathrm{d} \mathbf{x} \\
& +\varepsilon C_{4} \int_{\Omega} \varphi\left(\left|d_{h, k}^{+} \operatorname{div}\left(\xi^{2} d_{h, k}^{+} \mathbf{u}\right)\right|\right)+\varphi\left(\left|d_{h, k}^{-} \operatorname{div}\left(\xi^{2} d_{h, k}^{+} \mathbf{u}\right)\right|\right)+\varphi\left(\left|\operatorname{div}\left(\xi^{2} d_{h, k}^{+} \mathbf{u}\right)\right|\right) \mathrm{d} \mathbf{x} \\
\leq & c\left(\varepsilon^{-1}, C_{4},\|\xi\|_{2, \infty}\right) \int_{Q} \varphi(|\nabla \mathbf{u}|) \mathrm{d} \mathbf{x} \\
& +\varepsilon C_{4} c\left(\|\xi\|_{1, \infty}\right) \int_{\Omega} \varphi\left(\left|d_{h, k}^{+}\left(\xi d_{h, k}^{+} \mathbf{u}\right)\right|\right)+\varphi\left(\left|d_{h, k}^{-}\left(\xi d_{h, k}^{+} \mathbf{u}\right)\right|\right) \mathrm{d} \mathbf{x} \\
& c c\left(\varepsilon^{-1}, C_{4},\|\xi\|_{2, \infty}\right) \int_{Q} \varphi(|\nabla \mathbf{u}|) \mathrm{d} \mathbf{x}+\varepsilon C_{4} c\left(\|\xi\|_{1, \infty}\right) \int_{\Omega} \varphi\left(\xi\left|\nabla d_{h, k}^{+} \mathbf{u}\right|\right) \mathrm{d} \mathbf{x} .
\end{aligned}
$$


Finally, using inequality (2.1) with $\Delta_{2}(\varphi)<\infty$, estimate (2.4), Corollary 4.2(ii) and inequality (5.6), one shows that

$$
\begin{aligned}
\left|I_{4}\right| & \leq c \int_{Q} \varphi^{*}(|\mathbf{f}|) \mathrm{d} \mathbf{x}+c \int_{\Omega} \varphi\left(\left|d_{h, k}^{-} \mathbf{v}\right|\right) \mathrm{d} \mathbf{x} \\
& \leq c \int_{Q} \varphi^{*}(|\mathbf{f}|) \mathrm{d} \mathbf{x}+c \int_{Q} \varphi(|\nabla \mathbf{v}|) \mathrm{d} \mathbf{x} \\
& \leq c \int_{Q} \varphi^{*}(|\mathbf{f}|) \mathrm{d} \mathbf{x}+c \int_{Q} \varphi\left(\left|\operatorname{div}\left(\xi^{2} d_{h, k}^{+} \mathbf{u}\right)\right|\right) \mathrm{d} \mathbf{x} \\
& \leq c \int_{Q} \varphi^{*}(|\mathbf{f}|)+\varphi(|\nabla \mathbf{u}|) \mathrm{d} \mathbf{x} .
\end{aligned}
$$

Applying the obtained estimates of $\left|I_{j}\right|$ to (5.4), we get

$$
\begin{aligned}
& \int_{\Omega} \xi^{2}\left|d_{h, k}^{+} \mathbf{F}(\mathbf{D u})\right|^{2}+\varphi\left(\xi\left|\nabla d_{h, k}^{+} \mathbf{u}\right|\right) \mathrm{d} \mathbf{x} \\
& \leq c\left(\varepsilon^{-1}, C_{4},\|\xi\|_{2, \infty}\right) \int_{Q} \varphi^{*}(|\mathbf{f}|)+\varphi(|\nabla \mathbf{u}|) \mathrm{d} \mathbf{x}+\varepsilon c\left(C_{4},\|\xi\|_{1, \infty}\right) \int_{\Omega} \varphi\left(\left|\xi \nabla d_{h, k}^{+} \mathbf{u}\right|\right) \mathrm{d} \mathbf{x} .
\end{aligned}
$$

By choosing $\varepsilon$ sufficiently small, the last term on the right-hand side may be absorbed into the left-hand side of the inequality. Hence, we have proved

$$
\int_{\Omega} \xi^{2}\left|d_{h, k}^{+} \mathbf{F}(\mathbf{D u})\right|^{2} \mathrm{~d} \mathbf{x} \leq c\left(\|\xi\|_{2, \infty}\right) \int_{Q} \varphi^{*}(|\mathbf{f}|)+\varphi(|\nabla \mathbf{u}|) \mathrm{d} \mathbf{x} .
$$

In view of (2.5), (2.6), this implies the desired estimate (5.2).

Acknowledgements Open Access funding provided by Projekt DEAL. We would like to thank the referees for their helpful comments.

Open Access This article is licensed under a Creative Commons Attribution 4.0 International License, which permits use, sharing, adaptation, distribution and reproduction in any medium or format, as long as you give appropriate credit to the original author(s) and the source, provide a link to the Creative Commons licence, and indicate if changes were made. The images or other third party material in this article are included in the article's Creative Commons licence, unless indicated otherwise in a credit line to the material. If material is not included in the article's Creative Commons licence and your intended use is not permitted by statutory regulation or exceeds the permitted use, you will need to obtain permission directly from the copyright holder. To view a copy of this licence, visit http://creativecommons.org/licenses/by/4.0/.

\section{References}

1. Beirão da Veiga, H.: On the global regularity of shear thinning flows in smooth domains. J. Math. Anal. Appl. 349(2), 335-360 (2009)

2. Bennett, C., Sharpley, R.: Interpolation of operators. In: Pure and Applied Mathematics, vol. 129. Academic Press Inc, Boston, MA (1988)

3. Berselli, L.C., Diening, L., Růžička, M.: Existence of strong solutions for incompressible fluids with shear dependent viscosities. J. Math. Fluid Mech. 12(1), 101-132 (2010)

4. Berselli, L.C., Růžička, M.: Global regularity properties of steady shear thinning flows. J. Math. Anal. Appl. 450(2), 839-871 (2017)

5. Bogovskii, M.E.: Solution of the first boundary value problem for the equation of continuity of an incompressible medium. Dokl. Akad. Nauk. SSSR 248, 1037-1040 (1979). English transl. in Soviet Math. Dokl. 20, 1094-1098 (1979)

6. Bogovskii, M.E.: Solution of some vector analysis problems connected with operators div and grad. In: Trudy Seminar S.L. Sobolev, vol. 80, pp. 5-40. Akademia Nauk SSSR (1980) 
7. Calderón, A.P., Zygmund, A.: On singular integrals. Am. J. Math. 78, 289-309 (1956)

8. Crispo, F.: A note on the global regularity of steady flows of generalized Newtonian fluids. Port. Math. 66(2), 211-223 (2009)

9. Cruz-Uribe, D.V., Martell, J.M., Pérez, C.: Weights, extrapolation and the theory of Rubio de Francia. In: Operator Theory: Advances and Applications, vol. 215. Birkhäuser/Springer Basel AG, Basel (2011)

10. Diening, L., Růžička, M.: Calderón-Zygmund operators on generalized lebesgue spaces $L^{p(\cdot)}$ and problems related to fluid dynamics. J. Reine Angew. Math. 563, 197-220 (2003)

11. Diening, L., Ettwein, F.: Fractional estimates for non-differentiable elliptic systems with general growth. Forum Math. 20(3), 523-556 (2008)

12. Diening, L., Kreuzer, C.: Linear convergence of an adaptive finite element method for the $p$-Laplacian equation. SINUM 46, 614-638 (2008)

13. Diening, L., Růžička, M., Schumacher, K.: A decomposition technique for John domains. Ann. Acad. Sci. Fenn. Ser. A. I. Math. 35(1), 87-114 (2010)

14. Evans, L.C.: Partial differential equations. In: Graduate Studies in Mathematics, vol. 19. American Mathematical Society, Providence, RI (1998)

15. Fuchs, M., Seregin, G.: Variational Methods for Problems from Plasticity Theory and for Generalized Newtonian Fluids. Springer, Berlin (2000)

16. Galdi, G.P.: An introduction to the mathematical theory of the Navier-Stokes equations. Vol. I. In: Springer Tracts in Natural Philosophy, vol. 38. Springer, New York (1994)

17. Gallardo, D.: Orlicz spaces for which the Hardy-Littlewood maximal operator is bounded. Publ. Mat. 32(2), 261-266 (1988)

18. Grafakos, L.: Classical Fourier analysis. In: Graduate Texts in Mathematics, vol. 249, 3rd edn. Springer, New York (2014)

19. Málek, J., Růžička, M., Shelukhin, V.V.: Herschel-Bulkley fluids: existence and regularity of steady flows. Math. Models Methods Appl. Sci. 15(12), 1845-1861 (2005)

20. Pick, L., Kufner, A., John, O., Fučík, S.: Function spaces. In: De Gruyter Series in Nonlinear Analysis and Applications, vol. 14, Vol. 1, extended edn. Walter de Gruyter \& Co., Berlin (2013)

21. Rao, M.M., Ren, Z.D.: Theory of Orlicz spaces. In: Monographs and Textbooks in Pure and Applied Mathematics, vol. 146. Marcel Dekker Inc., New York (1991)

22. Růžička, M., Diening, L.: Non-Newtonian fluids and function spaces. In: Proceedings of the Nonlinear Analysis, Function Spaces and Applications, NAFSA 2006 Prague, vol. 8, pp. 95-144 (2007)

23. Schumacher, K.: The Navier-Stokes Equations with Low Regularity Data in Weighted Function Spaces. Ph.D. Thesis, FB Mathematik, Universität Darmstadt (2007)

24. Schumacher, K.: Solutions to the equation $\operatorname{div} u=f$ in weighted Sobolev spaces. In: Parabolic and Navier-Stokes Equations Part 2. Banach Center Publication, vol. 81, pp. 433-440. Institute of Mathematics of the Polish Academy of Sciences, Warsaw (2008)

25. Talenti, G.: Boundedness of minimizers. Hokkaido Math. J. 19(2), 259-279 (1990)

Publisher's Note Springer Nature remains neutral with regard to jurisdictional claims in published maps and institutional affiliations. 